\title{
Synthesis of a New Class of Triazole-Linked Benzoheterocycles via 1,3-Dipolar Cycloaddition
}

\author{
Fernanda C. G. Barbosa and Ronaldo N. de Oliveira* \\ Departamento de Química, Laboratório de Síntese de Compostos Bioativos - LSCB, Universidade Federal \\ Rural de Pernambuco, Rua Dom Manoel de Medeiros s/n, Dois Irmãos, 52171-900 Recife-PE, Brazil
}

\begin{abstract}
Uma nova série de derivados 1,2,3,-triazólicos foi sintetizada a partir de ftalimidas e alcinos terminais na presença de quantidade catalítica de CuI. O presente protocolo forneceu 1,2,3-triazóis em moderados a bons rendimentos (44-89\%).
\end{abstract}

\begin{abstract}
A new series of 1,2,3-triazole derivatives have been synthesized from phthalimides and terminal alkynes in the presence of a catalytic amount of $\mathrm{CuI}$. The present protocol affords 1,2,3-triazoles in moderate to good yields (44-89\%).
\end{abstract}

Keywords: benzoheterocycles, 1,2,3-triazole, phthalimide, copper-catalyst, cycloaddition

\section{Introduction}

The synthesis of small molecules libraries for biological screening has gained impetus in the scientific community. Among the different functional moieties employed for this purpose, one commonly used is the nitrogen-containing heterocyclic compounds, which exhibit diverse biological and pharmacological activities. Compounds containing such an aza-heterocycle were described as trypanocidal agents, ${ }^{1}$ glycogen phosphorylase inhibitors, ${ }^{2}$ antitumor, ${ }^{3}$ antiviral, ${ }^{4}$ antimicrobial agents, ${ }^{5}$ antimycobacterial, ${ }^{6}$ among others. ${ }^{7}$ In particular, two widely known classes have been subject of investigation by our research group, namely the benzoheterocyclic compounds and the triazoles. ${ }^{8}$ These compounds have received much attention from medicinal chemists, who search for a heterocyclic scaffold of drugs in pharmacology, so that many efforts have been made in the optimization of their preparation methods. ${ }^{8-11}$ Such compounds are versatile molecules and their range of applications is steadily increasing including, among others, the areas of carbohydrates, materials sciences and nanosciences. ${ }^{12-14}$ Still in this context and without loss of structural simplicity, we have been attracted by the idea of designing compounds built from different heterocyclic blocks, seeking for an enhanced biological activity. In this work, we performed the synthesis of novel compounds based on 1,2,3-triazoles-linked benzoheterocycles. In

*e-mail: ronaldonoliveira@dq.ufrpe.br order to reach this goal, we employed the 1,3-dipolar cycloaddition reaction (1,3-DCRs) between an azide function and a terminal alkyne via a cross-linking process to afford the corresponding 1,2,3-triazoles, using a copper(I)-based catalyst, specifically CuI. It is worth to note that, in most cases explored in this work, there was no need of any additional base in the reaction medium as well as of any ligands for the catalytic system. ${ }^{15,16}$ The selected benzoheterocyclic alkynes are $S$-propargyl derivatives of benzimidazole-2-thiol (1a), benzothiazole2-thiol (1b) and benzoxazole-2-thiol (1c), $N$-propargyl derivatives of benzimidazole (1d) and phthalimide (1e). $N$-(3-Azidopropyl- and 4-azidobutyl) phthalimides (2a,b) were chosen as the phthalimide block. Furthermore, we have used a retrosynthetic strategy to obtain the desired products BTP (benzoheterocycle-1,2,3-triazolephthalimide), as shown in Figure 1.

To our knowledge, these three-block conjugations were not yet reported in the literature, which is surprising, in view of the potential ready availability and the growing impact of the triazole chemistry on organic synthesis. ${ }^{16}$

\section{Results and Discussion}

The starting materials (1a-e) were prepared via nucleophilic substitution between propargyl bromide $\left(\mathrm{BrCH}_{2} \mathrm{C} \equiv \mathrm{CH}\right)$ and the benzoheterocyclic compounds in the presence of $\mathrm{K}_{2} \mathrm{CO}_{3}$. This protocol afforded the corresponding terminal alkynes (1a-e) in $44-83 \%$ yields. 

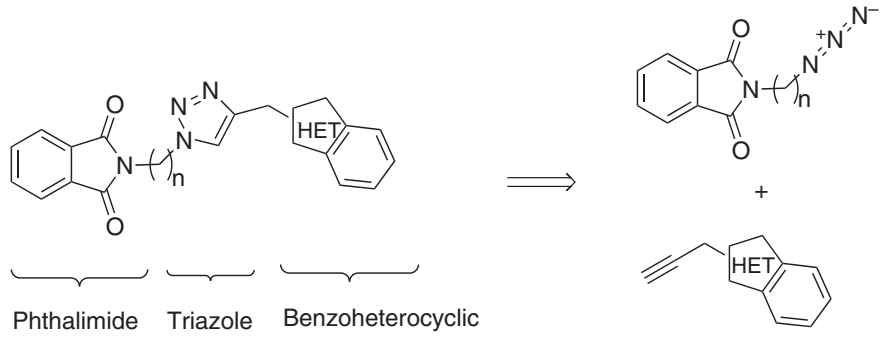

Figure 1. Retrosynthetic strategy of three-heterocyclic blocks conjugation.

The IR spectra of these compounds showed typical bands at 2112-2121 $\mathrm{cm}^{-1}$, corresponding to $\mathrm{C} \equiv \mathrm{C}$, and at $3176-3273 \mathrm{~cm}^{-1}$ to $\mathrm{C} \equiv \mathrm{C}-\mathrm{H}$ stretching related to the alkyne. The starting $N$-(azido-alkyl)phthalimide (2a) and (2b) were prepared from $N$-(bromoalkyl)phthalimides by reaction with $\mathrm{NaN}_{3}$ in $\mathrm{DMF}$ at $60{ }^{\circ} \mathrm{C}$ during $24 \mathrm{~h}$. This procedure afforded the azido-compounds (2a, , b) as white solids which were used without any purification.

In order to perform the cycloaddition reaction towards three-heterocyclic blocks sequence BTP, we used the most common cycloaddition protocol between $\mathrm{N}$-(4azidobutyl)-phthalimide (2b) and the acetylene (1d) using $\mathrm{Cu}(\mathrm{OAc})_{2}$ as $\mathrm{Cu}(\mathrm{II})$ source and sodium ascorbate in media of tert-BuOH: $\mathrm{H}_{2} \mathrm{O} 50 \%$ at room temperature. ${ }^{10}$ However, this methodology has provided low yields ( $c a .23 \%$ ).

Looking at this result, we turned to the following modified protocol. The copper-catalyzed condensation of 2-propargylsulfanylbenzothiazole (1) 1.5 mol equiv.) with azide (2b, 1 mol equiv.) in $\mathrm{CH}_{2} \mathrm{Cl}_{2}$ at $30{ }^{\circ} \mathrm{C}$ afforded 1,2,3-triazole (4b) in $84 \%$ yield after column chromatography (Table 1, entry 8 ). Encouraged by this positive result, we have focused our effort on the synthesis of new derivatives of 1-[ $N$-phthalimidoalkyl]-4-heteroaryl$1 H$-1,2,3-triazoles (3) and (4) using this protocol (Scheme 1 and Table 1).

Reaction of azidoalkylphthalimides (2a or $\mathbf{2 b}$ ) with benzoheterocycles (1a-e) provides easy conversion to the corresponding 1,2,3-triazoles (3) and (4) in 44-89\% yield after column chromatography or recrystallization (Table 1). To our knowledge, such a cross-linking reaction between alkyne and azide groups, using $\mathrm{CuI}$ as catalyst in dichloromethane and in the absence of base, has not been performed yet. In only one case, the addition of a base, namely $\mathrm{Et}_{3} \mathrm{~N}$, was necessary to reduce the reaction time (Table 1, entry 11).

In order to test the applicability of the method $\left(\mathrm{CuI} / \mathrm{CH}_{2} \mathrm{Cl}_{2} / \mathrm{rt}\right)$ to a different substrate rather than the benzoheterocyclic moiety, a different kind of functionality was investigated. In this respect, we examined the condensation reaction between $N$-3-azido-propyl (2a) or $N$-4-azidobutylphthalimide (2b) and the ethynyl ketone (1f), and checked the ready formation of 1,2,3-triazoles (3f) and (4f) in excellent yields $92 \%$ and $91 \%$, respectively (Table 1, entries 6 and 12). These results are in agreement with the literature, which can be justified by the fact that $\alpha$-carbonyl-alkynes are highly reactive. ${ }^{16}$

In summary, we have developed a convenient route, with easy work up, for the synthesis of a new class of 1,2,3-triazole derivatives (3) and (4) in moderate to good yields (44-92\%). This new tris-heterocyclic sequence (benzoheterocycles-triazole-phthalimide, BTP) represents a set of potentially interesting compounds for biological activity screening and we believe that applications for them will be soon found in organic and medicinal chemistry.

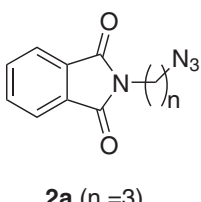

$\mathbf{2 a}(\mathrm{n}=3)$
$\mathbf{2 b}(\mathrm{n}=4)$

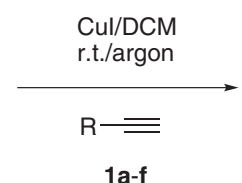

1a-f

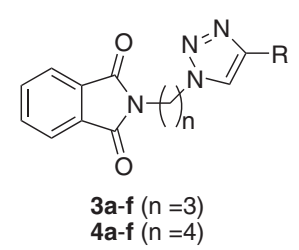

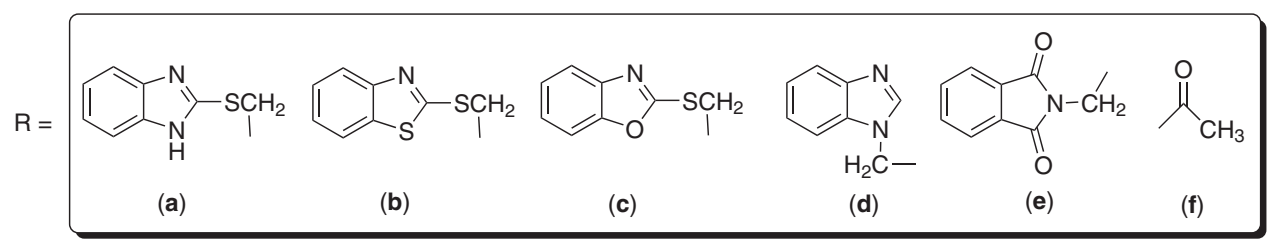

Scheme 1. Synthesis of the compounds 1,2,3-triazoles (3a-f) and (4a-f). 
Table 1. Cu-Catalyzed formation of (3a-f) and (4a-f)

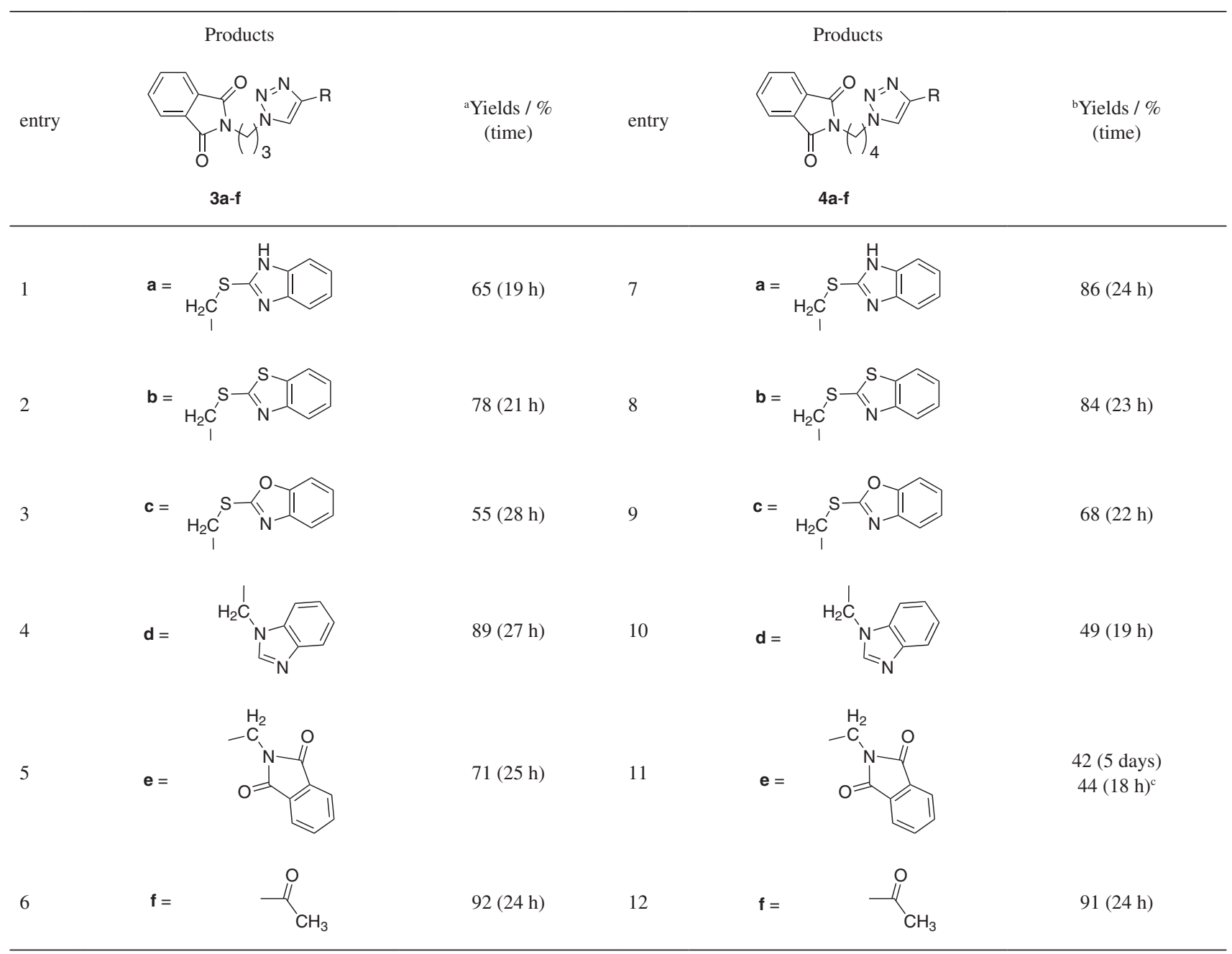

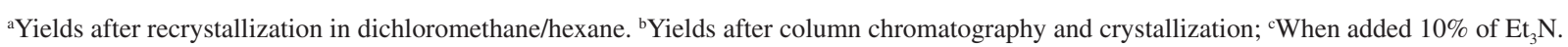

\section{Experimental}

All commercially available reagents were used as received. All organic solvents used for the synthesis were of analytical grade. Column chromatography was performed on Merck silica gel 60 (70-230 mesh). All reactions were monitored by TLC analysis contained $\mathrm{GF}_{254}$. IR spectra were recorded on a IFS66 Bruker spectrophotometer using $\mathrm{KBr}$ discs. ${ }^{1} \mathrm{H}$ and ${ }^{13} \mathrm{C}$ NMR spectra were obtained on Varian unity plus-300, 400 and 500 spectrometer using tetramethylsilane as internal reference. Elemental analyses were carried out on a EA1110 CHNS-O analyzer. High resolution mass spectra HRMS were recorded on a Shimadzu Liquid Chrom MS LCMS-IT-TOF using acetonitrile or methanol as solvent. Air- and moisturesensitive reactions were performed under inert atmosphere of argon. Melting points were determined on a PFM II BioSan apparatus and are uncorrected.
Typical procedure for the synthesis of 1,4-disubstituted 1,2,3-triazoles

In a round-bottom flask, the azide-compound (2a) (100 mg, $0.43 \mathrm{mmol}, 1$ equiv. of azide function) was charged with the terminal alkyne (1a) (113 mg, $0.60 \mathrm{mmol}$, 1.4 equiv.), the solvent ( $5 \mathrm{~mL}$ of $\mathrm{DCM})$ and the copper catalyst based on either CuI $10 \mathrm{~mol} \%$ (12 mg, $0.063 \mathrm{mmol}$ each according to the alkyne-compound). The reaction was performed by stirring at room temperature under argon atmosphere, during 18-28 $\mathrm{h}$. The resulting mixture was washed with $\mathrm{NH}_{4} \mathrm{OH}$ and then extracted with dichloromethane. The combined organic layers were dried with over anhydrous sodium sulfate and then the solvent was removed in vacuum. Purification of the crude material by column chromatography using hexane-EtOAc (8:2) as eluent or recrystallization in $\mathrm{CH}_{2} \mathrm{Cl}_{2}$ /hexane mixture afforded the title compound (3a). 
4-(Benzimidazol-2-ylsulfanyl)methyl-1-(3-phthalimidopropyl)-1,2,3-triazole (3a)

Yield 65\%; mp 102-103 ${ }^{\circ} \mathrm{C} ; \mathrm{R}_{\mathrm{f}}=0.5\left(\mathrm{CH}_{2} \mathrm{Cl}_{2}\right.$-EtOAc, 9:1); IR $v_{\text {max }} / \mathrm{cm}^{-1}$ : 3452, 3140, 2940, 1766, 1706, 1464, 1430, 1396, 1359, 996, 750, 717. ${ }^{1} \mathrm{H}$ NMR (300 MHz, $\mathrm{CDCl}_{3}$ ): $\delta 2.28\left(\mathrm{q}, 2 \mathrm{H}, \mathrm{CH}_{2}\right), 2.90(\mathrm{bs}, 1 \mathrm{H}, \mathrm{NH}), 3.69(\mathrm{t}, 2 \mathrm{H}, J 6.6 \mathrm{~Hz}$, $\mathrm{NCH}_{2}$ ), 4.35 (t, $\left.2 \mathrm{H}, J 6.9 \mathrm{~Hz}, \mathrm{NCH}_{2}\right), 4.67$ (s, 2H, $\mathrm{SCH}_{2}$ ), 7.29 (t, $\left.1 \mathrm{H}, J 7.5 \mathrm{~Hz}, \mathrm{H}_{\text {arom }}\right), 7.41\left(\mathrm{t}, 1 \mathrm{H}, J 7.5 \mathrm{~Hz}, \mathrm{H}_{\text {arom }}\right.$ ), 7.71-7.84 (m, 6H, Phth, NCH=, $\left.\mathrm{H}_{\text {arom }}\right), 7.92(\mathrm{~d}, 1 \mathrm{H}, J 8.1 \mathrm{~Hz}$, $\left.\mathrm{H}_{\text {arom }}\right) .{ }^{13} \mathrm{C}$ NMR $\left(75.5 \mathrm{MHz}, \mathrm{CDCl}_{3}\right): \delta 27.7,29.3,34.9$, 47.9, 121.1, 121.5, 123.4, 124.3, 126.0, 131.8 (C in triazole), $134.2(\mathrm{NCH}=$ in triazole $), 135.4,143.6,152.8$ and $165.9(\mathrm{C}$ in benzimidazole), 168.2 ( $\mathrm{C}=\mathrm{O}$ in phthalimide). $\mathrm{m} / \mathrm{z} \mathrm{LC}-\mathrm{MS}$ $\left[\mathrm{M}\left(\mathrm{C}_{21} \mathrm{H}_{18} \mathrm{~N}_{6} \mathrm{O}_{2} \mathrm{~S}\right)+\mathrm{H}\right]^{+}$calc.: 419.1290; found: 419.2774 .

4-(Benzothiazol-2-ylsulfanyl)methyl-1-(3-phthalimidopropyl)-1,2,3-triazole (3b)

Yield 78\%; mp 103-105 ${ }^{\circ} \mathrm{C} ; \mathrm{R}_{\mathrm{f}}=0.4\left(\mathrm{CH}_{2} \mathrm{Cl}_{2}\right.$-EtOAc, 9:1); IR $v_{\max } / \mathrm{cm}^{-1}: 3140,2940,1767,1706,1464,1430$, $1397,1360,994,717 .{ }^{1} \mathrm{H} \mathrm{NMR}\left(300 \mathrm{MHz}, \mathrm{CDCl}_{3}\right): \delta 2.27$ (q, $\left.2 \mathrm{H}, \mathrm{CH}_{2}\right), 3.69$ (t, J $\left.6.6 \mathrm{~Hz}, 2 \mathrm{H}, \mathrm{NCH}_{2}\right), 4.34(\mathrm{t}, J 6.9 \mathrm{~Hz}, 2 \mathrm{H}$, $\mathrm{NCH}_{2}$ ), $4.66\left(\mathrm{~s}, 2 \mathrm{H}, \mathrm{SCH}_{2}\right), 7.29$ (ddd, $1 \mathrm{H}, J 7.5,7.5,1.2 \mathrm{~Hz}$, $\mathrm{H}_{\text {arom }}$ ), 7.42 (ddd, $1 \mathrm{H}, J$ 7.5, 7.5, $1.2 \mathrm{~Hz}, \mathrm{H}_{\text {arom }}$ ), 7.72-7.77 (dd, $3 \mathrm{H}, J$ 5.4, 3.0, Phth and $\left.\mathrm{H}_{\text {arom }}\right), 7.81-7.85$ (m, 3H, Phth and $\mathrm{NCH}=), 7.91\left(\mathrm{dd}, 1 \mathrm{H}, J 7.5 \mathrm{~Hz}, \mathrm{H}_{\text {arom }}\right) \cdot{ }^{13} \mathrm{C} \mathrm{NMR}(75.5 \mathrm{MHz}$, $\left.\mathrm{CDCl}_{3}\right): \delta 27.6,29.3,34.8,47.8,121.0,121.5,123.3,124.3$, $125.9,131.7$ (C in triazole), 134.1, 135.4, 143.7, $152.9\left(\mathrm{C}_{\text {arom }}\right.$ in benzothiazole), 165.8, 168.2 ( $\mathrm{C}=\mathrm{O}$ in phthalimide). Anal. Calc. $\mathrm{C}_{21} \mathrm{H}_{17} \mathrm{~N}_{5} \mathrm{O}_{2} \mathrm{~S}$ : C, 57.91; H, 3.93; N, 16.08; S, 14.73. Found: C, 58.18; H, 4.03; N, 15.93; S, 14.58.

4-(Benzoxazol-2-ylsulfanyl)methyl-1-(3-phthalimidopropyl)-1,2,3-triazole (3c)

Yield 55\%; mp 131-133 ${ }^{\circ} \mathrm{C} ; \mathrm{R}_{\mathrm{f}}=0.6\left(\mathrm{CH}_{2} \mathrm{Cl}_{2}\right.$-EtOAc, 9:1); IR $v_{\text {max }} / \mathrm{cm}^{-1}: 3150,2939,1765,1709,1495,1454$, 1433, 1397, 1362, 1216, 1134, 715. 'H NMR (400 MHz, $\left.\mathrm{CDCl}_{3}\right): \delta 2.28\left(\mathrm{q}, 2 \mathrm{H}, \mathrm{CH}_{2}\right), 3.69\left(\mathrm{t}, 2 \mathrm{H}, J 6.3 \mathrm{~Hz}, \mathrm{NCH}_{2}\right.$ ), 4.35 (t, $2 \mathrm{H}, J 6.9 \mathrm{~Hz}, \mathrm{NCH}_{2}$ ), 4.61 (s, $\left.2 \mathrm{H}, \mathrm{SCH}_{2}\right), 7.19-7.29$ $\left(\mathrm{m}, 2 \mathrm{H}, \mathrm{H}_{\text {arom }}\right), 7.43\left(\mathrm{dd}, 1 \mathrm{H}, J 7.5,1.5 \mathrm{~Hz}, \mathrm{H}_{\text {arom }}\right), 7.62(\mathrm{~d}$, $1 \mathrm{H}, J 7.2 \mathrm{~Hz}, \mathrm{H}_{\text {arom }}$ ), 7.70-7.75 (dd, 2H, J 5.7, 3.0, Phth), 7.78-7.84 (dd, 2H, J 5.1, 3.0, Phth), 7.93 (s, 1H, NCH=). ${ }^{13} \mathrm{C} \mathrm{NMR}\left(100 \mathrm{MHz}, \mathrm{CDCl}_{3}\right): \delta 26.7,29.3,34.8,47.8,109.9$ ( $\mathrm{C}_{\text {arom }}$ in benzoxazole $), 118.4$ ( $\mathrm{C}_{\text {arom }}$ in benzoxazole $), 123.3$, $123.9,124.2,131.8$ ( $\mathrm{C}$ in triazole), $134.2(\mathrm{NCH}=$ in triazole $)$, 141.7, 152.0, 164.3, 168.2 (C=O in phthalimide). $m / z$ LC-MS $\left[\mathrm{M}\left(\mathrm{C}_{21} \mathrm{H}_{17} \mathrm{~N}_{5} \mathrm{O}_{3} \mathrm{~S}\right)+\mathrm{H}\right]^{+}$calc.: 420.1130 . Found: 420.1027.

4-(Benzimidazolemethyl)-1-(3-phthalimidopropyl)-1,2,3triazole $(3 \boldsymbol{d})$

Yield 89\%; mp $148-150{ }^{\circ} \mathrm{C} ; \mathrm{R}_{\mathrm{f}}=0.2\left(\mathrm{CH}_{2} \mathrm{Cl}_{2}\right.$-EtOAc, 9:1); IR $v_{\text {max }} / \mathrm{cm}^{-1}: 3141,3089,3050,3020,2943,1769$,
1703, 1614, 1399, 1042, 722. ${ }^{1} \mathrm{H}$ NMR (300 MHz, $\left.\mathrm{CDCl}_{3}\right)$ : $\delta 2.26\left(\mathrm{q}, 2 \mathrm{H}, \mathrm{CH}_{2}\right), 3.66\left(\mathrm{t}, 2 \mathrm{H}, J 6.8 \mathrm{~Hz}, \mathrm{NCH}_{2}\right), 4.34$ (t, $\left.2 \mathrm{H}, J 7.2 \mathrm{~Hz}, \mathrm{NCH}_{2}\right), 5.49\left(\mathrm{~s}, 2 \mathrm{H}, \mathrm{NCH}_{2}\right), 7.28(\mathrm{~m}, 2 \mathrm{H}$, $\left.\mathrm{H}_{\text {arom }}\right), 7.64$ (s, 1H, N=CH in benzimidazole), 7.67-7.71 (m, $\left.5 \mathrm{H}, \mathrm{Phth}, \mathrm{H}_{\text {arom }}\right), 7.79\left(\mathrm{~s}, 1 \mathrm{H}, \mathrm{NCH}=\right.$ in triazole). ${ }^{13} \mathrm{C} \mathrm{NMR}$ $\left(75.5 \mathrm{MHz}, \mathrm{CDCl}_{3}\right): \delta 29.2,34.7,47.8,104.9,110.0,122.5$, $122.9,123.3,123.1,131.7$ ( $\mathrm{C}$ in triazole), $134.1(\mathrm{NCH}=$ in triazole), 142.4, 168.2 ( $\mathrm{C}=\mathrm{O}$ in phthalimide). Anal. Calc. $\mathrm{C}_{21} \mathrm{H}_{18} \mathrm{~N}_{6} \mathrm{O}_{2}\left(2.5 \mathrm{H}_{2} \mathrm{O}\right): \mathrm{C}, 58.46 ; \mathrm{H}, 5.37$. Found: C, 57.79; $\mathrm{H}, 4.66$.

4-(N-Phthalimidomethyl)-1-(3-phthalimidopropyl)-1,2,3triazole (3e)

Yield 71\% (Lit. $\left.{ }^{17} 96 \%\right) ; \mathrm{R}_{\mathrm{f}}=0.4\left(\mathrm{CH}_{2} \mathrm{Cl}_{2}\right.$-EtOAc, 9:1); IR $v_{\text {max }} / \mathrm{cm}^{-1}: 3589,3150,2953,1767,1706,1467,1429$, 1396, 714. ${ }^{1} \mathrm{H}$ NMR (300 MHz, $\mathrm{CDCl}_{3}$ ): $\delta 2.29$ (q, $2 \mathrm{H}$, $\mathrm{CH}_{2}$ ), 3.73 (t, $2 \mathrm{H}, J 6.3 \mathrm{~Hz}, \mathrm{NCH}_{2}$ ), $4.36(\mathrm{t}, 2 \mathrm{H}, J 7.2 \mathrm{~Hz}$, $\left.\mathrm{NCH}_{2}\right), 4.97\left(\mathrm{~s}, 2 \mathrm{H}, \mathrm{PhthCH}_{2}\right), 7.68-7.75(\mathrm{~m}, 5 \mathrm{H}, \mathrm{NCH}=$ and Phth), 7.80-7.85 (m, 4H, Phth). ${ }^{13} \mathrm{C}$ NMR (75.5 MHz, $\left.\mathrm{CDCl}_{3}\right): \delta 29.4,32.9,34.9,47.9,123.3,123.4,131.8,132.0$, 134.0, 134.2, 167.6 and 168.3 ( $\mathrm{C}=\mathrm{O}$ in phthalimide). $\mathrm{m} / \mathrm{z}$ LC-MS $\left[\mathrm{M}\left(\mathrm{C}_{22} \mathrm{H}_{18} \mathrm{~N}_{5} \mathrm{O}_{4}\right)+\mathrm{H}\right]^{+}$calc.: 416.1359. Found: 416.1305 .

\section{4-Acetyl-1-(3-phthalimidopropyl)-1,2,3-triazole (3f)}

Yield 92\%; mp 188-190 ${ }^{\circ} \mathrm{C} ; \mathrm{R}_{\mathrm{f}}=0.7$ (Hexane-EtOAc, 1:1); IR $v_{\max } / \mathrm{cm}^{-1}: 3106,3048,1776,1701,1680,1384$, 1177, 1030, 721. ${ }^{~} \mathrm{H}$ NMR (300 MHz, $\left.\mathrm{CDCl}_{3}\right): \delta 2.37$ (q, $\left.2 \mathrm{H}, \mathrm{CH}_{2}\right), 2.66\left(\mathrm{~s}, 3 \mathrm{H}, \mathrm{COCH}_{3}\right), 3.76(\mathrm{t}, J 6.9 \mathrm{~Hz}, 2 \mathrm{H}$, $\left.\mathrm{NCH}_{2}\right), 4.46$ (t, J 6.9 Hz, 2H, $\mathrm{NCH}_{2}$ ), 7.73-7.77 (dd, J 3.3, 5.7, 2H, Phth), 7.84-7.87 (dd, J 3.3, 5.4, 2H, Phth), 8.26 (s, $1 \mathrm{H}, \mathrm{NCH}=)$. NMR ${ }^{13} \mathrm{C}\left(75.5 \mathrm{MHz}, \mathrm{CDCl}_{3}\right): \delta 27.2,29.3$, 34.7, 48.2, 123.5, 131.7 (C in triazole), $134.3(\mathrm{NCH}=$ in triazole), $168.2\left(\mathrm{C}=\mathrm{O}\right.$ in phthalimide), $192.7\left(\mathrm{COCH}_{3}\right)$. $\mathrm{m} / \mathrm{z}$ LC-MS $\left[\mathrm{M}\left(\mathrm{C}_{15} \mathrm{H}_{14} \mathrm{~N}_{4} \mathrm{O}_{3}\right)+\mathrm{H}\right]^{+}$calc.: 299.1144 . Found: 299.1163.

4-(Benzimidazol-2-ylsulfanyl)methyl-1-(4-phthalimidobutyl)-1,2,3-triazole (4a)

Yield 86\%; mp 100-102 ${ }^{\circ} \mathrm{C} ; \mathrm{R}_{\mathrm{f}}=0.6$ (Hexane-EtOAc, 7:3); IR $v_{\max } / \mathrm{cm}^{-1}: 3460,3145,3053,2938,2871,1765$, 1705, 1459, 1429, 1399, 1372, 1337, 1308, 1222, 1045, 996, 920, 747, 723. ${ }^{1} \mathrm{H}$ NMR (400 MHz, $\mathrm{CDCl}_{3}$ ): $\delta 1.67$ (q, $2 \mathrm{H}, \mathrm{CH}_{2}$ ), 1.90 (q, 2H, $\mathrm{CH}_{2}$ ), 2.81 (bs, $1 \mathrm{H}, \mathrm{NH}$ ), 3.68 (t, $\left.2 \mathrm{H}, J 5.4 \mathrm{~Hz}, \mathrm{NCH}_{2}\right), 4.35$ (t, $2 \mathrm{H}, J 5.4 \mathrm{~Hz}, \mathrm{NCH}_{2}$ ), 4.69 (s, $\left.2 \mathrm{H}, \mathrm{SCH}_{2}\right), 7.29\left(\mathrm{t}, 1 \mathrm{H}, J 6.0 \mathrm{~Hz}, \mathrm{H}_{\text {arom }}\right), 7.41(\mathrm{t}, 1 \mathrm{H}$, $\left.J 5.7 \mathrm{~Hz}, \mathrm{H}_{\text {arom }}\right), 7.64(\mathrm{~s}, 1 \mathrm{H}, \mathrm{NCH}=), 7.69-7.71(\mathrm{dd}, 2 \mathrm{H}$, $J$ 2.4, 4.2, Phth), 7.74 (d, $1 \mathrm{H}, J 5.7 \mathrm{~Hz}, \mathrm{H}_{\text {arom }}$ ), 7.78-7.82 (dd, 2H, J 2.4, 3.6, Phth), 7.90 (d, 1H, J $6.0 \mathrm{~Hz}, \mathrm{H}_{\text {arom }}$ ). ${ }^{13} \mathrm{C} \mathrm{NMR}\left(100 \mathrm{MHz}, \mathrm{CDCl}_{3}\right): \delta 25.5,27.3,27.7,36.7,49.5$, $121.0,121.4,123.2,124.3,126.0,131.8$ (C in triazole), 
$133.9(\mathrm{NCH}=$ in triazole $), 135.4,143.8,152.9$ and 165.8 ( $\mathrm{C}$ in benzimidazole), 168.3 ( $\mathrm{C}=\mathrm{O}$ in phthalimide). Anal. Calc.: $\mathrm{C}_{22} \mathrm{H}_{20} \mathrm{~N}_{6} \mathrm{O}_{2} \mathrm{~S} \cdot 0.8 \mathrm{H}_{2} \mathrm{O}: \mathrm{C}, 59.13 ; \mathrm{H}, 4.87$. Found: $\mathrm{C}$, 58.68; H, 4.33 .

4-(Benzothiazol-2-ylsulfanyl)methyl-1-(4-phthalimidobutyl)-1,2,3-triazole (4b)

Yield 84\%; mp 92-94 ${ }^{\circ} \mathrm{C} ; \mathrm{R}_{\mathrm{f}}=0.6\left(\mathrm{CH}_{2} \mathrm{Cl}_{2}\right.$-EtOAc, 9:1); IR $v_{\max } / \mathrm{cm}^{-1}: 2972,2854,1770,1710,1613,1465$, 1428, 1397, 1304, 1041, 757, 719. 'H NMR (300 MHz, $\left.\mathrm{CDCl}_{3}\right): \delta 1.68\left(\mathrm{q}, 2 \mathrm{H}, \mathrm{CH}_{2}\right), 1.91\left(\mathrm{q}, 2 \mathrm{H}, \mathrm{CH}_{2}\right), 3.68(\mathrm{t}$, $\left.2 \mathrm{H}, J 6.9 \mathrm{~Hz}, \mathrm{NCH}_{2}\right), 4.38\left(\mathrm{t}, 2 \mathrm{H}, J 7.2 \mathrm{~Hz}, \mathrm{NCH}_{2}\right), 4.70$ (s, $\left.2 \mathrm{H}, \mathrm{SCH}_{2}\right), 7.30\left(\mathrm{dd}, 1 \mathrm{H}, J 7.5,7.5 \mathrm{~Hz}, \mathrm{H}_{\text {arom }}\right), 7.42$ (ddd, $1 \mathrm{H}, J$ 7.7, 7.7 Hz, $\mathrm{H}_{\text {arom }}$ ), 7.69-7.78 (m, 4H, $\mathrm{NCH}=$, Phth, $\left.\mathrm{H}_{\text {arom }}\right), 7.80-7.83$ (m, 2H, Phth), 7.92 (d, 1H, J 7.5 Hz, $\left.\mathrm{H}_{\text {arom }}\right) .{ }^{13} \mathrm{C} \mathrm{NMR}\left(75.5 \mathrm{MHz}, \mathrm{CDCl}_{3}\right): \delta 22.6,25.4,27.3$, 27.3, 36.7, 109.9, 116.0, 118.4, 121.1, 122.4, 123.3, 129.5, $131.8,134.0,143.9,155.2,168.3(\mathrm{C}=\mathrm{O}$ in phthalimide). Anal. Calc. $\mathrm{C}_{22} \mathrm{H}_{19} \mathrm{~N}_{5} \mathrm{O}_{2} \mathrm{~S}_{2}$ : C, 58.78; H, 4.26; N, 15.58; S, 14.27. Found: C, 58.21; H, 4.43; N, 15.04; S, 14.27. $\mathrm{m} / \mathrm{z}$ LC-MS $[\mathrm{M}+\mathrm{H}]^{+}$calc.: 450.1058 . Found: 450.1085 .

4-(Benzoxazol-2-ylsulfanyl)methyl-1-(4-phthalimidobutyl)1,2,3-triazole $(\mathbf{4 c})$

Yield 67\%; mp 68-70 ${ }^{\circ} \mathrm{C} ; \mathrm{R}_{\mathrm{f}}=0.8\left(\mathrm{CH}_{2} \mathrm{Cl}_{2}\right.$-EtOAc, 9:1); IR $v_{\max } / \mathrm{cm}^{-1}: 3467,3147,3053,2933,2867,1773$, 1716, 1613, 1502, 1454, 1397, 1365, 1236, 1131, 1096, $1042,740,721 .{ }^{~} \mathrm{H}$ NMR $\left(400 \mathrm{MHz}, \mathrm{CDCl}_{3}\right): \delta 1.70(\mathrm{q}, 2 \mathrm{H}$, $\mathrm{CH}_{2}$ ), 1.97 (q, 2H, $\left.\mathrm{CH}_{2}\right), 3.69$ (t, $2 \mathrm{H}, J 7.2 \mathrm{~Hz}, \mathrm{NCH}_{2}$ ), 4.35 (t, $2 \mathrm{H}, J 7.2 \mathrm{~Hz}, \mathrm{NCH}_{2}$ ), $4.64\left(\mathrm{~s}, 2 \mathrm{H}, \mathrm{SCH}_{2}\right), 7.22-7.7 .30$ $\left(\mathrm{m}, 2 \mathrm{H}, \mathrm{H}_{\text {arom }}\right), 7.44\left(\mathrm{~d}, 1 \mathrm{H}, J 7.6 \mathrm{~Hz}, \mathrm{H}_{\text {arom }}\right), 7.62(\mathrm{~d}, 1 \mathrm{H}, J$ $7.6 \mathrm{~Hz}, \mathrm{H}_{\text {arom }}$ ), 7.70-7.73 (dd, 2H, J 5.6, 3.2, Phth), 7.75 (s, $1 \mathrm{H}, \mathrm{NCH}=), 7.79-7.82$ (dd, $2 \mathrm{H}, J$ 5.6, 3.2, Phth). ${ }^{13} \mathrm{C} \mathrm{NMR}$ $\left(100 \mathrm{MHz}, \mathrm{CDCl}_{3}\right): \delta 25.5,26.6,27.3,36.7,49.8,110.0$, $118.4,123.3,124.1,124.4,131.9$ (C in triazole), 134.0 $(\mathrm{NCH}=$ in triazole $), 141.7,152.0,159.7,168.3(\mathrm{C}=\mathrm{O}$ in phthalimide). Anal. Calc. $\mathrm{C}_{22} \mathrm{H}_{19} \mathrm{~N}_{5} \mathrm{O}_{3} \mathrm{~S} \cdot\left(0.4 \mathrm{H}_{2} \mathrm{O}\right)$ : C, 59.96; H, 4.53; N, 15.89; S, 7.27. Found: C, 59.31; H, 4.45; N, 15.59; S, 7.92.

4-(Benzimidazolemethyl)-1-(4-phthalimidobutyl)-1,2,3triazole $(\mathbf{4 d})$

Yield 49\%; mp 103-105 ${ }^{\circ} \mathrm{C} ; \mathrm{R}_{\mathrm{f}}=0.3\left(\mathrm{CH}_{2} \mathrm{Cl}_{2}\right.$-EtOAc, 9:1); IR $v_{\max } / \mathrm{cm}^{-1}: 3137,3089,2937,1769,1708,1494$, 1458, 1399, 1042, 747, 720. ${ }^{1} \mathrm{H} \mathrm{NMR}\left(300 \mathrm{MHz}, \mathrm{CDCl}_{3}\right)$ : $\delta 1.65\left(\mathrm{q}, 2 \mathrm{H}, \mathrm{CH}_{2}\right), 1.87\left(\mathrm{q}, 2 \mathrm{H}, \mathrm{CH}_{2}\right), 3.65(\mathrm{t}, 2 \mathrm{H}$, $J 6.9 \mathrm{~Hz}, \mathrm{NCH}_{2}$ in triazole), $4.32\left(\mathrm{t}, 2 \mathrm{H}, J 7.2 \mathrm{~Hz}, \mathrm{NCH}_{2}\right.$ in phthalimide), $5.52\left(\mathrm{~s}, 2 \mathrm{H}, \mathrm{NCH}_{2}\right), 7.26-7.31\left(\mathrm{~m}, 3 \mathrm{H}, \mathrm{H}_{\text {arom }}\right)$, $7.49(\mathrm{~s}, 1 \mathrm{H}, \mathrm{NCH}=$ in benzimidazole $), 7.68-7.72(\mathrm{~m}, 2 \mathrm{H}$, dd, 2H, J 5.4, 3.0, Phth), 7.70-7.83 (m, 3H, Phth, $\mathrm{H}_{\text {arom }}$ ), $8.32(\mathrm{~s}, 1 \mathrm{H}, \mathrm{NCH}=$ in triazole $) .{ }^{13} \mathrm{C}$ NMR-APT $(125.6 \mathrm{MHz}$,
$\left.\mathrm{CDCl}_{3}\right): \delta 25.4,27.3,36.7,40.7,49.6,109.9,120.4,122.0$, $122.4,123.2,123.3,131.9$ (C in triazole), $134.1(\mathrm{NCH}=$ in triazole), $143.0,168.3$ (C=O in phthalimide). $\mathrm{m} / 2$ LC-MS $\left[\left(\mathrm{C}_{22} \mathrm{H}_{20} \mathrm{~N}_{6} \mathrm{O}_{2}\right)+\mathrm{H}\right]^{+}$calc.: 401.1728. Found: 401.1714.

4-(N-Phthalimidomethyl)-1-(4-phthalimidobutyl)-1,2,3triazole $(4 \mathrm{e})$

Yield 44\%; mp 150-151 ${ }^{\circ} \mathrm{C} ; \mathrm{R}_{\mathrm{f}}=0.45\left(\mathrm{CH}_{2} \mathrm{Cl}_{2}\right.$-EtOAc, 9:1); IR $v_{\text {max }} / \mathrm{cm}^{-1}: 3133,2952,1768,1702,1614,1467$, 1428, 1397, 1089, 1030, 939, 715. 'H NMR (400 MHz, $\left.\mathrm{CDCl}_{3}\right): \delta 1.70\left(\mathrm{q}, 2 \mathrm{H}, \mathrm{CH}_{2}\right), 1.92\left(\mathrm{q}, 2 \mathrm{H}, \mathrm{CH}_{2}\right), 3.70(\mathrm{t}$, $2 \mathrm{H}, J 6.6 \mathrm{~Hz}, \mathrm{NCH}_{2}$ in triazole), 4.37 (t, $2 \mathrm{H}, J 6.9 \mathrm{~Hz}$, $\mathrm{NCH}_{2}$ in phthalimide), $4.98\left(\mathrm{~s}, 2 \mathrm{H}, \mathrm{PhthCH}_{2}\right), 7.63(\mathrm{~s}, 1 \mathrm{H}$, $\mathrm{NCH}=)$, 7.68-7.73 (m, 4H, Phth), 7.79-7.86 (m, 4H, Phth). ${ }^{13} \mathrm{C} \mathrm{NMR}\left(100 \mathrm{MHz}, \mathrm{CDCl}_{3}\right): \delta 25.5,27.4,32.9,36.8,49.6$, $123.3,123.4,131.8,132.0$ (C in triazole), $134.0(\mathrm{NCH}=$ in triazole), 167.6 and 168.3 ( $\mathrm{C}=\mathrm{O}$ in phthalimide). Anal. Calc. $\mathrm{C}_{23} \mathrm{H}_{19} \mathrm{~N}_{5} \mathrm{O}_{4} \cdot\left(1 / 2 \mathrm{H}_{2} \mathrm{O}\right): \mathrm{C}, 63.01 ; \mathrm{H}, 4.60 ; \mathrm{N}, 15.97$. Found: C, 63.11; H, 4.09; N, 15.98 .

\section{4-Acetyl-1-(4-phthalimidobutyl)-1,2,3-triazole (4f)}

Yield 91\%; mp 151-153 ${ }^{\circ} \mathrm{C} ; \mathrm{R}_{\mathrm{f}}=0.6\left(\mathrm{CH}_{2} \mathrm{Cl}_{2}\right.$-EtOAc, 9:1); IR $v_{\text {max }} / \mathrm{cm}^{-1}: 3138,2923,2851,1709,1681,1537$, $1400,1216,1035,723 .{ }^{1} \mathrm{H}$ NMR $\left(500 \mathrm{MHz}, \mathrm{CDCl}_{3}\right): \delta 1.72$ (q, $\left.2 \mathrm{H}, \mathrm{CH}_{2}\right), 1.96\left(\mathrm{q}, 2 \mathrm{H}, \mathrm{CH}_{2}\right), 2.66$ (s, 3H, $\left.\mathrm{COCH}_{3}\right), 3.73$ (t, $J 6.5 \mathrm{~Hz}, 2 \mathrm{H}, \mathrm{NCH}_{2}$ in triazole), $4.45(\mathrm{t}, J 6.5 \mathrm{~Hz}, 2 \mathrm{H}$, $\mathrm{NCH}_{2}$ in phthalimide), 7.70-7.72 (m, 2H, Phth), 7.82-7.84 (m, 2H, Phth), 8.04 (s, 1H, NCH=). ${ }^{13} \mathrm{C}$ NMR-APT (125.6 $\left.\mathrm{MHz}, \mathrm{CDCl}_{3}\right): \delta 25.5,27.1\left(\mathrm{CH}_{3}\right), 27.3,36.7,49.9,123.4$ $\left(\mathrm{CH}_{\text {arom }}\right), 132.0(\mathrm{C}$ in triazole $), 134.1(\mathrm{NCH}=$ in triazole $)$, 168.4 $\left(\mathrm{C}=\mathrm{O}\right.$ in phthalimide), $192.9\left(\mathrm{COCH}_{3}\right)$. Anal. Calc. for $\mathrm{C}_{16} \mathrm{H}_{16} \mathrm{~N}_{4} \mathrm{O}_{3}: \mathrm{C}, 61.53 ; \mathrm{H}, 5.16 ; \mathrm{N}, 17.94$. Found: $\mathrm{C}$, 61.89; H, 5.22; N, 17.64. $\mathrm{m} / z$ L C-MS $\left[\mathrm{M}\left(\mathrm{C}_{16} \mathrm{H}_{16} \mathrm{~N}_{4} \mathrm{O}_{3}\right)+\right.$ $\mathrm{H}]^{+}$calc.: 313.1301 . Found: 313.1305.

\section{Supplementary Information}

Supplementary data are available free of charge at http://jbcs.sbq.org.br, as PDF file.

\section{Acknowledgments}

The authors are grateful to Pernambuco State Foundation of Science and Technology (FACEPE: APQ-0776-1.06/08) for financial support and CNPq/PIBIC/UFRPE-Brazil for providing a fellowship to one of us (F. C. G. B.). Our thanks are also due to Central Analytical CENAPESQ-UFRPE for facilities installation and DQF-UFPE for obtaining the spectra data. We thank to Dr. Helcio José Batista for his continuous support throughout the preparation of the manuscript. 


\section{References}

1. da Silva, E. N., Jr.; de Moura, M. A. B. F.; Pinto, A. V.; Pinto, M. C. F. R.; de Souza, M. C. B. V.; Araújo, A. J.; Pessoa, C.; Costa-Lotufo, L. V.; Montenegro, R. C.; de Moraes, M. O.; Ferreira, V. F.; Goulart, M. O. F.; J. Braz. Chem. Soc. 2009, 20, 635.

2. Bokor, E.; Docsa, T.; Gergely, P.; Somsák, L.; Bioorg. Med. Chem. 2010, 18, 1171.

3. Yang, X.-L.; Xu, C.-M.; Lin, S.-M.; Chen, J.-X.; Ding, J.-C.; Wu, H.-Y.; Su, W.-K.; J. Braz. Chem. Soc. 2010, 21, 37; Kok, S. H. L.; Gambari, R.; Chui, C. H.; Yuen, M. C. W.; Lin, E.; Wong, R. S. M.; Lau, F. Y.; Cheng, G. Y. M.; Lam, W. S.; Chan, S. H.; Lam, K. H.; Cheng, C. H.; Lai, P. B. S.; Yu, M. W. Y.; Cheung, F.; Tang, J. C. O.; Chan, A. S. C.; Bioorg. Med. Chem. 2008, 16, 3626.

4. Pessoa-Mahana, D.; Núñez, A.; Espinosa, C.; Mella-Raipán, J.; Pessoa-Mahana, H.; J. Braz. Chem. Soc. 2010, 21, 63; Jordão, A. K.; Afonso, P. P.; Ferreira, V. F.; de Souza, M. C. B. V.; Almeida, M. C. B.; Beltrame, C. O.; Paiva, D. P.; Wardell, S. M. S. V.; Wardell, J. L.; Tiekink, E. R. T.; Damaso, C. R.; Cunha, A. C.; Eur. J. Med. Chem. 2009, 44, 3777.

5. Rohini, R.; Shanker, K.; Reddy, P. M.; Ravinder, V.; J. Braz. Chem. Soc. 2010, 21, 49.

6. Gallardo, H.; Conte, G.; Bryk, F.; Lourenço, M. C. S.; Costa, M. S.; Ferreira, V. F.; J. Braz. Chem. Soc. 2007, 18, 1285.

7. Anegundi, R. I.; Puranik, V. G.; Hotha, S.; Org. Biomol. Chem. 2008, 6, 779 .

8. de Oliveira, R. N.; Sinou, D.; Srivastava, R. M.; J. Carbohydr. Chem. 2006, 25, 407; de Oliveira, R. N.; Sinou, D.; Srivastava, R. M.; Synthesis 2006, 467; de Oliveira, R. N.; Mendonça Jr., F. J. B.; Sinou, D.; de Melo, S. J.; Srivastava, R. M.; Synlett 2006, 3049.
9. dos Anjos, J. V.; Sinou, D.; de Melo, S. J.; Srivastava, R. M.; Carbohydr. Res. 2007, 342, 2440; Gouin, S. G.; Bultel, L.; Falentin, C.; Kovensky, J.; Eur. J. Org. Chem. 2007, 7, 1160; Wilkinson, B. L.; Bornaghi, L. F.; Poulsen, S.-A.; Houston, T. A.; Tetrahedron 2006, 62, 8115; Katritzky, A. R.; Rachwal, S.; Chem. Rev. 2010, 110, 1564.

10. Huisgen, R.; Pure Appl. Chem. 1989, 61, 613; Alvarez, R.; Velázquez, S.; San-Félix, A.; Aquaro, S.; De Clercq, E.; Perno, C. -F.; Karlsson, A.; Balzarine, J.; Camarasa, M. J.; J. Med. Chem. 1994, 37, 4185; Tornøe, C. W.; Christensen, C.; Meldal, M.; J. Org. Chem. 2002, 67, 3057; Rostovtsev, V. V.; Green, L. G.; Fokin, V. V.; Sharpless, K. B.; Angew. Chem., Int. Ed. 2002, 41, 2596.

11. Rodionov, V. O.; Presolski, S. I.; Gardinier, S.; Lim, Y.-H.; Finn, M. G.; J. Am. Chem. Soc. 2007, 129, 12696.

12. Alix, A.; Chassaing, S.; Pale, P.; Sommer, J.; Tetrahedron 2008, 64, 8922.

13. Alonso, F.; Moglie, Y.; Radivoy, G.; Yus, M.; Tetrahedron Lett. 2009, 50, 2358; Sharghi, H.; Khalifeh, R.; Doroodmand, M. M.; Adv. Synth. Catal. 2009, 351, 207.

14. Dururgkar, K. A.; Gonnade, R. G.; Ramana, C. V.; Tetrahedron 2009, 65, 3974; Steinmetz, V.; Couty, F.; David, O. R. P.; Chem. Commun. 2009, 343; Whiting, M.; Tripp, J. C.; Lin, Y. -C.; Lindstrom, W.; Olson, A. J.; Elder, J. H.; Sharpless, K. B.; Fokin, V. V.; J. Med. Chem. 2006, 49, 7697; Fournier, D.; Prez, F. D.; Macromolecules 2008, 41, 4622.

15. Díez-González, S.; Nolan, S. P. Angew. Chem., Int. Ed. 2008, 47, 8881 .

16. Meldal, M.; Tornøe, C. W.; Chem. Rev. 2008, 108, 2952.

17. Kacprzak, K.; Synlett 2005, 6, 943.

Submitted: June 21, 2010

Published online: October 21, 2010 


\title{
Synthesis of a New Class of Triazole-Linked Benzoheterocycles via 1,3-Dipolar Cycloaddition
}

\author{
Fernanda C. G. Barbosa and Ronaldo N. de Oliveira* \\ Departamento de Química, Universidade Federal Rural de Pernambuco, Rua Dom Manoel de \\ Medeiros S/N, Dois Irmão, 52171-030 Recife-PE, Brazil
}

Synthesis of terminal alkynes (1a-e)

$1 \mathrm{mmol}$ of benzoheterocyclic and $1 \mathrm{mmol}$ of $\mathrm{K}_{2} \mathrm{CO}_{3}$ were suspended in anhydrous DMF ( $5 \mathrm{~mL})$. Then, 1.5 equiv. of propargyl bromide ( $80 \%$ solution in toluene) was added. The reaction mixture was stirred for $20 \mathrm{~h}$ at room temperature. The mixture was then extracted with dichloromethane/water. The combined organic layers were dried over sodium sulfate anhydrous and concentrated under reduced pressure, and the residue was purified by chromatography on silica gel (hexane:EtOAc, 7:3) to afford the corresponding propargylic benzoheterocycles (1a-e).

Synthesis of N-3-(azidopropyl)phthalimide (2a) or N-4-(azidobutyl)phthalimide (2b)

$N$-(bromoalkyl)phthalimide ( $500 \mathrm{mg}$ ) in $2.5 \mathrm{~mL}$ of DMF was charged in a round-bottom flask. Then, 1.5 equiv. of sodium azide was introduced and the reaction mixture was allowed to stir at $60{ }^{\circ} \mathrm{C}$ for $24 \mathrm{~h}$ under argon atmosphere. The mixture was then cooled to room temperature and extraction with dichloromethane was done. The combined organic layers were dried over sodium sulfate anhydrous and concentrated under reduced pressure.

\section{N-3-(azidopropyl)phthalimide (2a)}

Yield 75\%; white solid; IR $v_{\max } / \mathrm{cm}^{-1}: 2945,2100$ $\left(\mathrm{N}_{3}\right), 1711(\mathrm{C}=\mathrm{O}), 1399,1040,723 .{ }^{1} \mathrm{H} \mathrm{NMR}(300 \mathrm{MHz}$, CDCl3): $\delta 1.96(\mathrm{q}, 2 \mathrm{H}), 3.38(\mathrm{t}, 2 \mathrm{H}, J 6.9 \mathrm{~Hz}), 3.79(\mathrm{t}, 2 \mathrm{H}$, $J 6.9 \mathrm{~Hz}), 7.73(\mathrm{dd}, 2 \mathrm{H}, J 5.7$ and $3.0 \mathrm{~Hz}), 7.86(\mathrm{dd}, 2 \mathrm{H}$, $J 5.7$ and $3.0 \mathrm{~Hz}$ ).

\section{N-4-(azidobutyl)phthalimide (2b)}

Yield 61\%; white solid; IR $v_{\max } / \mathrm{cm}^{-1}: 2950,2096\left(\mathrm{~N}_{3}\right)$, $1709(\mathrm{C}=\mathrm{O}), 1396,719 .{ }^{1} \mathrm{H}$ NMR (300 MHz, CDCl3): $\delta$ $1.65(\mathrm{~m}, 2 \mathrm{H}), 1.78(\mathrm{~m}, 2 \mathrm{H}), 3.33(\mathrm{t}, 2 \mathrm{H}, J 6.6 \mathrm{~Hz}), 3.79(\mathrm{t}$, $2 \mathrm{H}, J 6.9 \mathrm{~Hz}), 7.72(\mathrm{dd}, 2 \mathrm{H}, J 5.7$ and $3.0 \mathrm{~Hz}), 7.85(\mathrm{dd}$, $2 \mathrm{H}, \mathrm{J} 5.7$ and $3.0 \mathrm{~Hz}$ ).

*e-mail: ronaldonoliveira@dq.ufrpe.br 

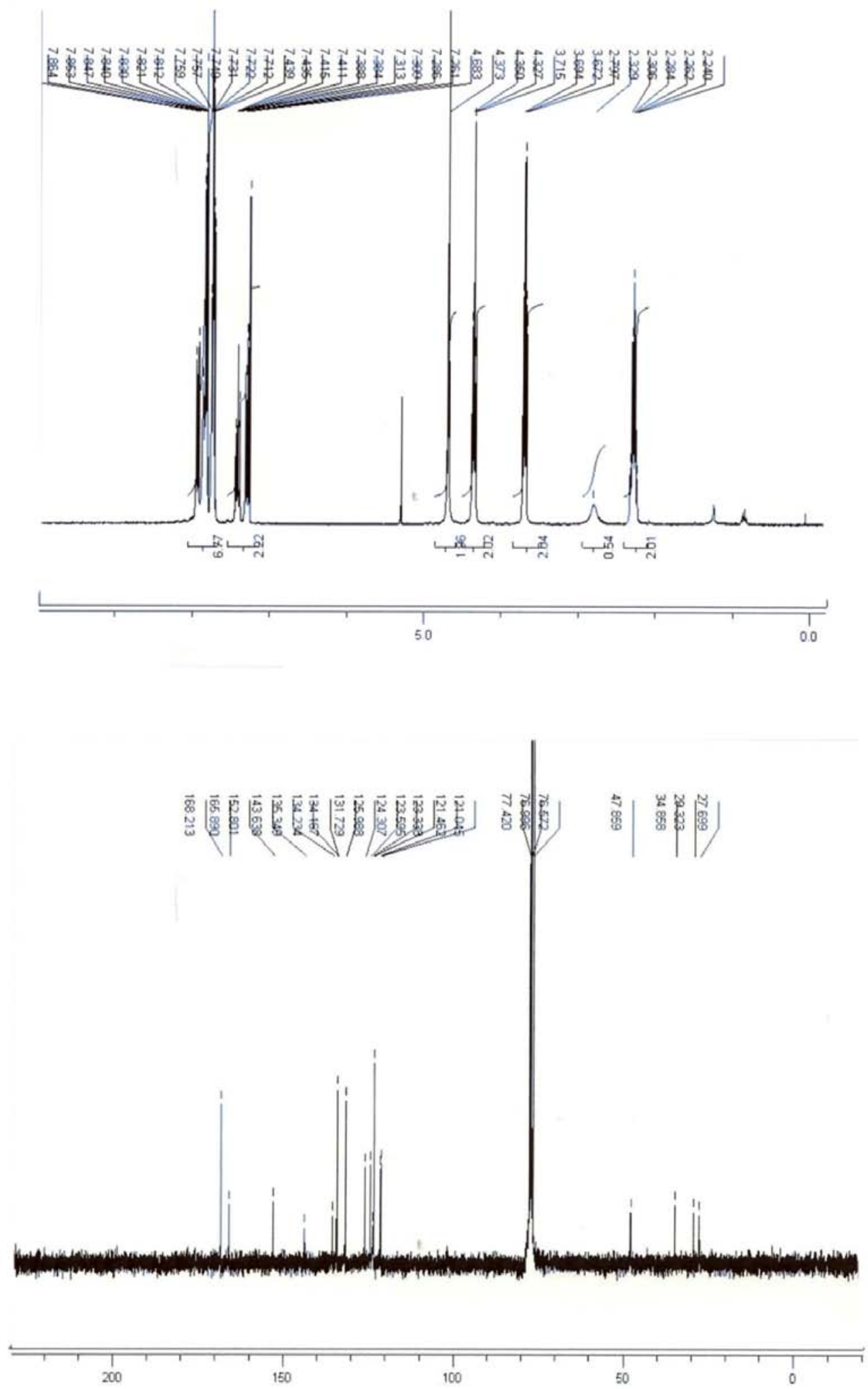

Figure S1. ${ }^{1} \mathrm{H}(300 \mathrm{MHz})$ and ${ }^{13} \mathrm{C}(75.5 \mathrm{MHz}) \mathrm{NMR}$ spectrum of compound 3a in $\mathrm{CDCl}_{3}$. 


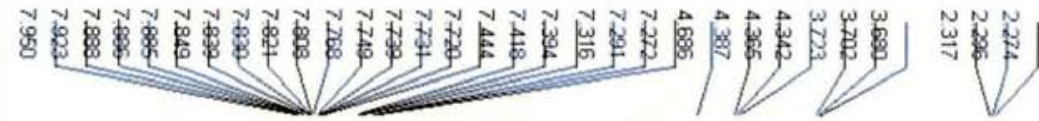
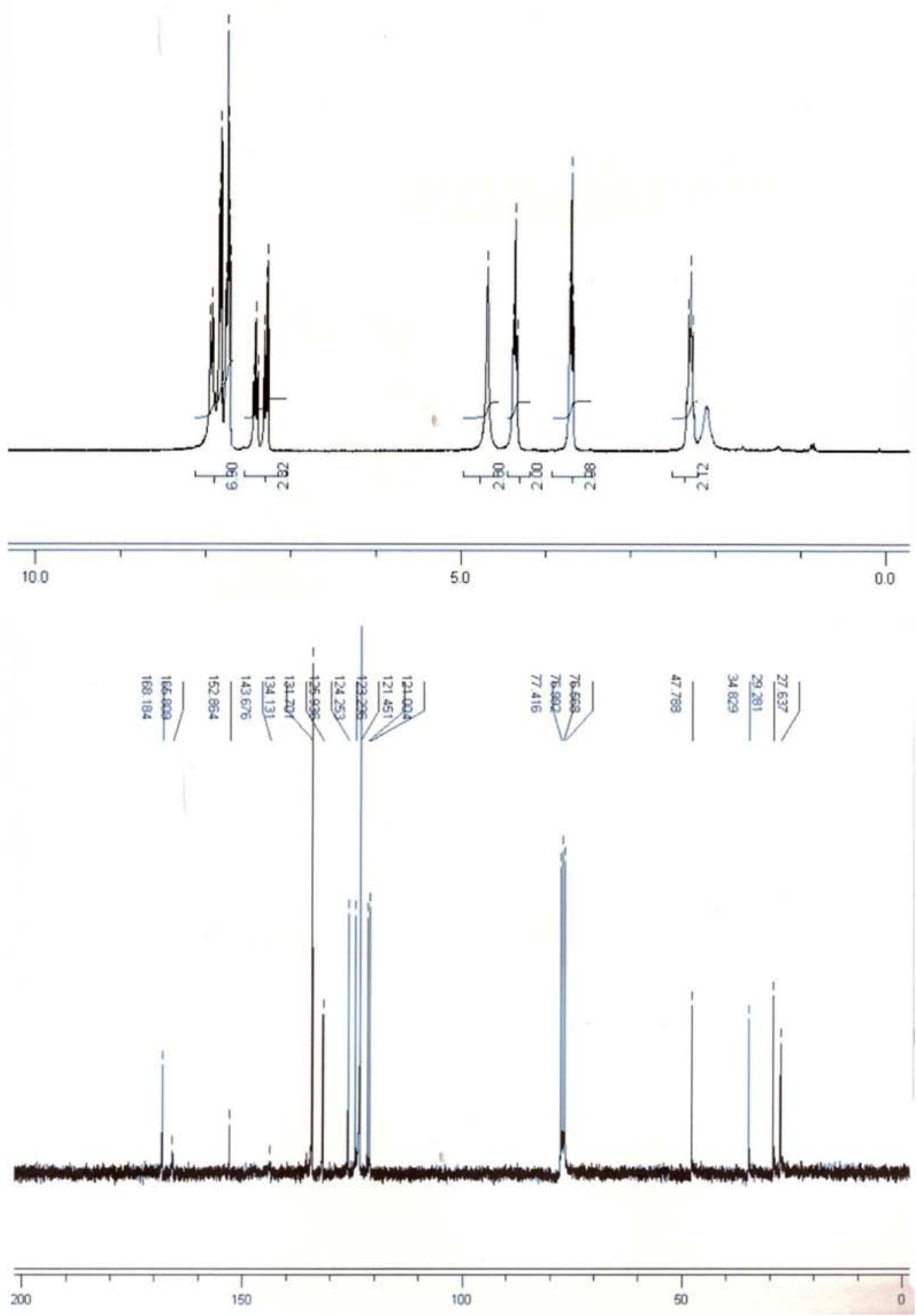

Figure S2. ${ }^{1} \mathrm{H}(300 \mathrm{MHz})$ and ${ }^{13} \mathrm{C}(75 \mathrm{MHz}) \mathrm{NMR}$ spectrum of compound $\mathbf{3 b}$ in $\mathrm{CDCl}_{3}$. 

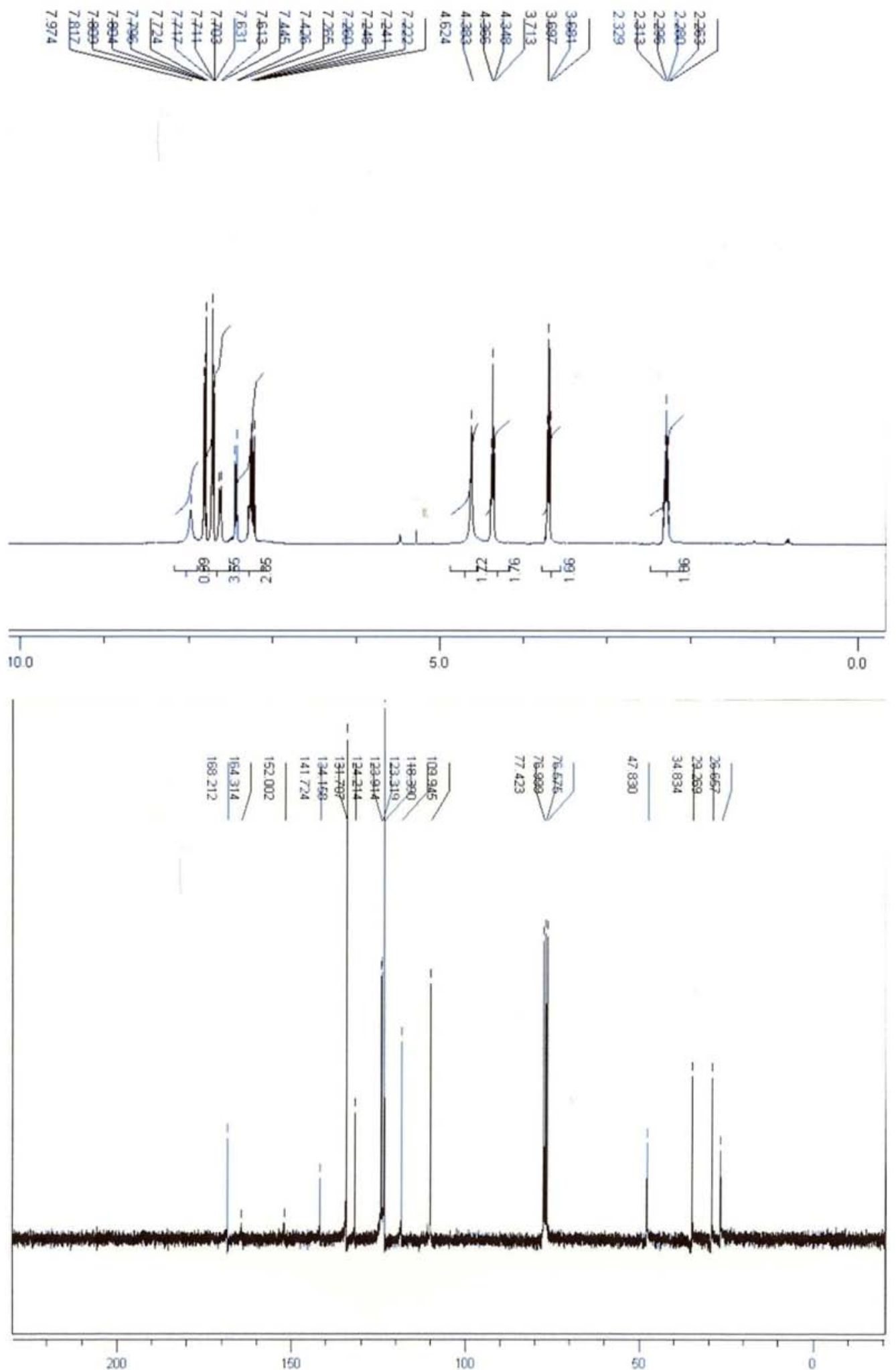

Figure S3. ${ }^{1} \mathrm{H}(400 \mathrm{MHz})$ and ${ }^{13} \mathrm{C}(100 \mathrm{MHz}) \mathrm{NMR}$ spectrum of compound $\mathbf{3 c}$ in $\mathrm{CDCl}_{3}$. 

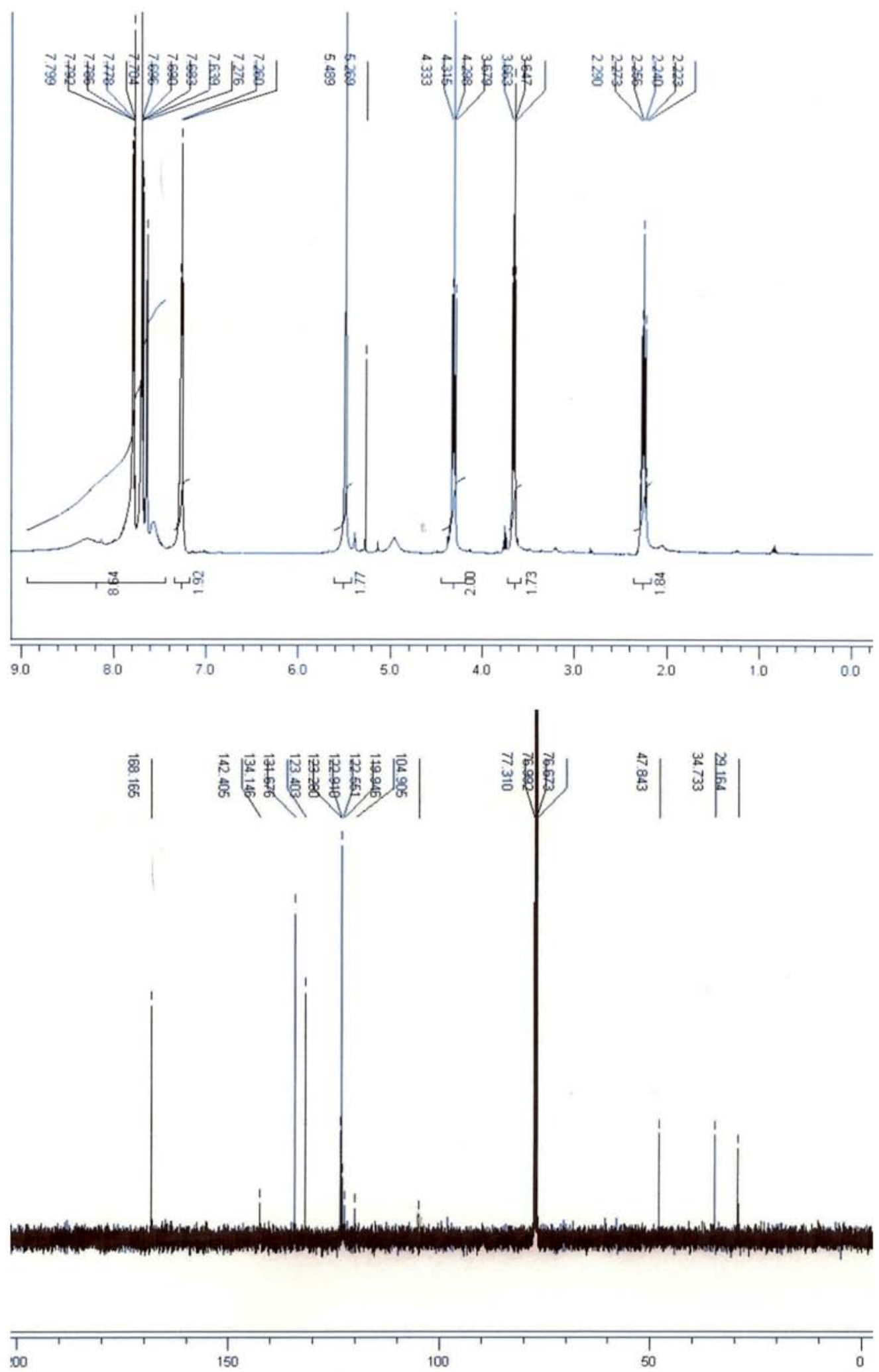

Figure S4. ${ }^{1} \mathrm{H}(400 \mathrm{MHz})$ and ${ }^{13} \mathrm{C}(100 \mathrm{MHz}) \mathrm{NMR}$ spectrum of compound $\mathbf{3 d}$ in $\mathrm{CDCl}_{3}$. 

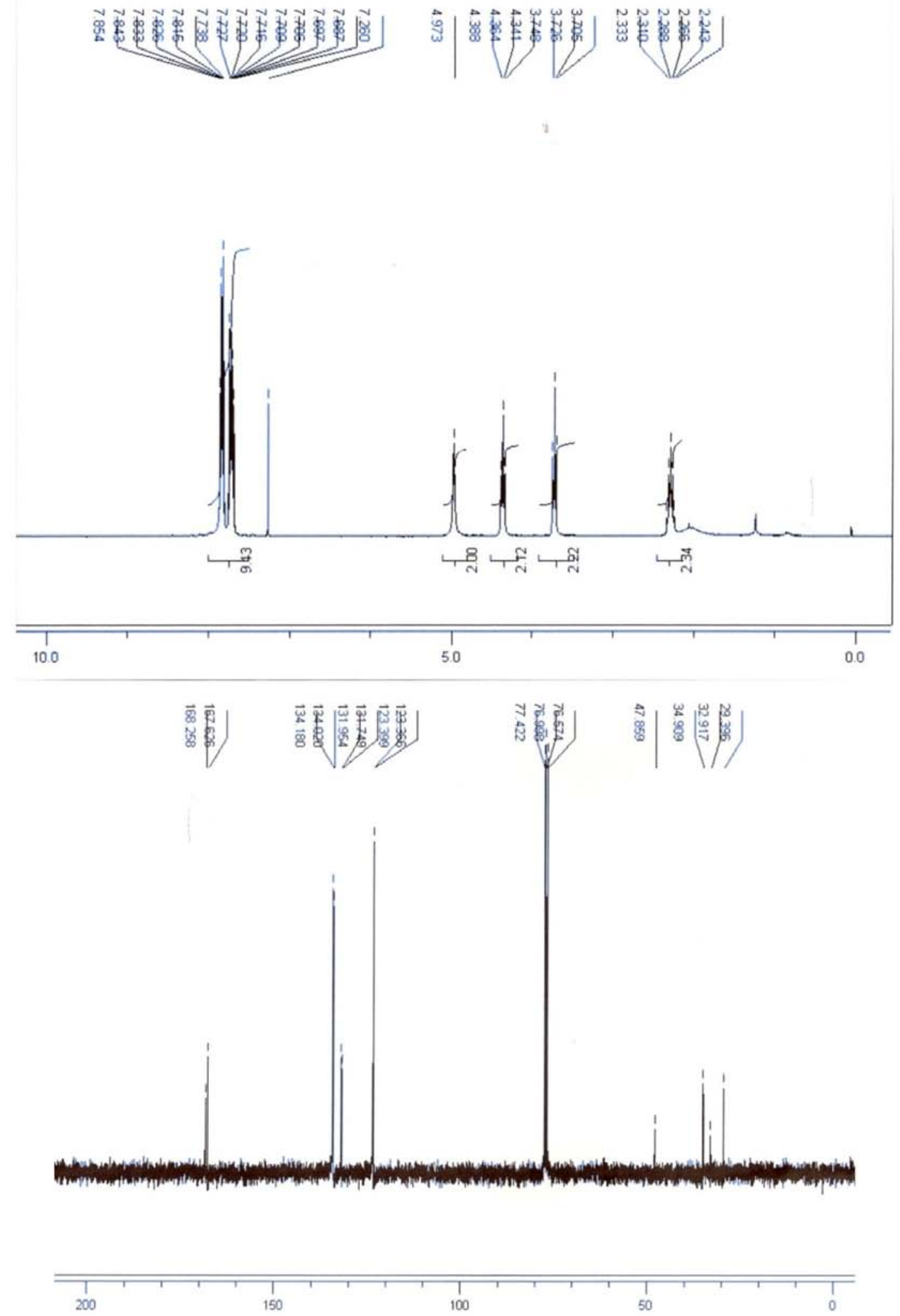

Figure S5. ${ }^{1} \mathrm{H}(300 \mathrm{MHz})$ and ${ }^{13} \mathrm{C}(75.5 \mathrm{MHz}) \mathrm{NMR}$ spectrum of compound $\mathbf{3 e}$ in $\mathrm{CDCl}_{3}$. 

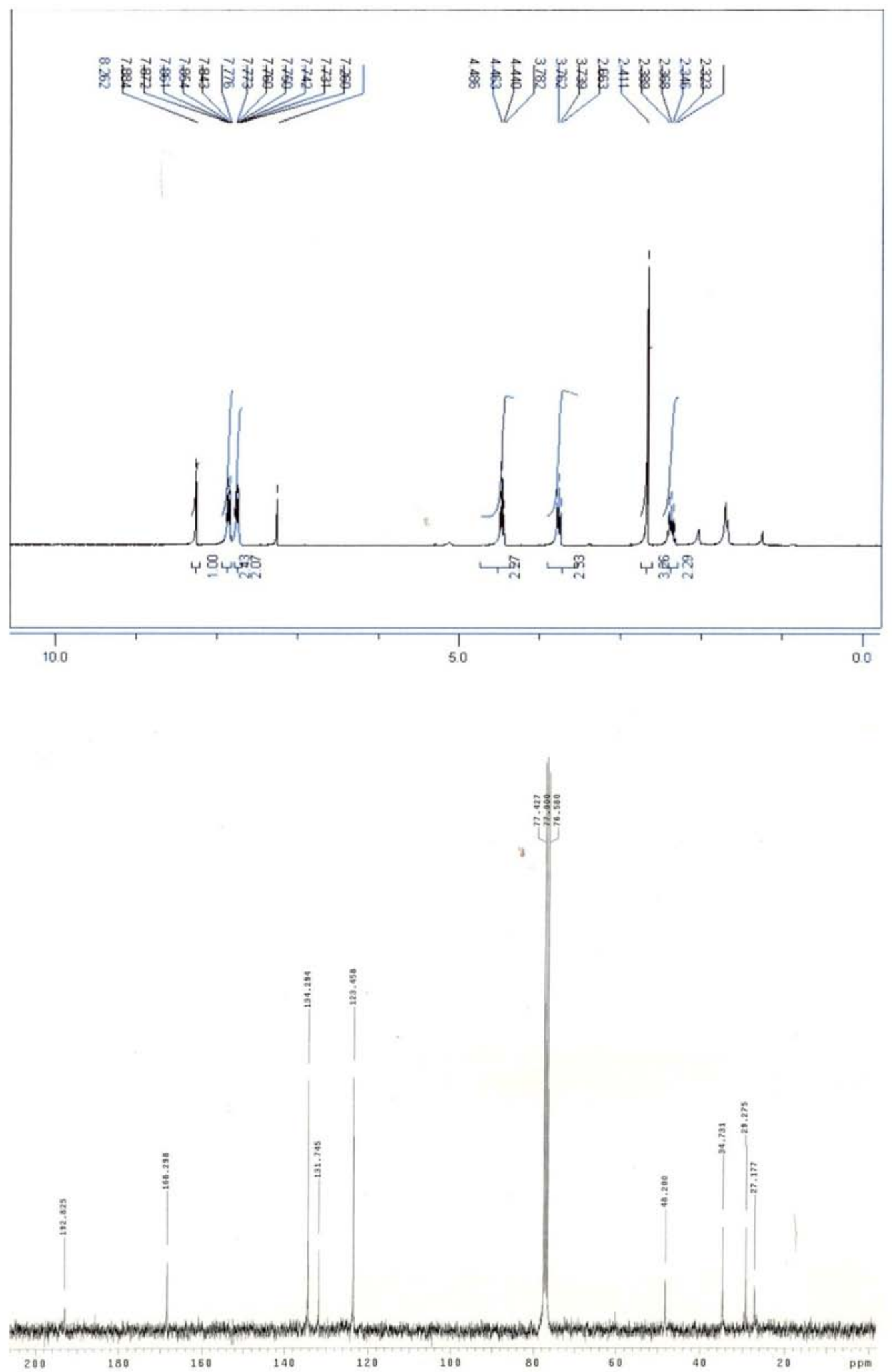

Figure S6. ${ }^{1} \mathrm{H}(300 \mathrm{MHz})$ and ${ }^{13} \mathrm{C}(75.5 \mathrm{M} \mathrm{Hz}) \mathrm{NMR}$ spectrum of compound $\mathbf{3 f}$ in $\mathrm{CDCl}_{3}$. 

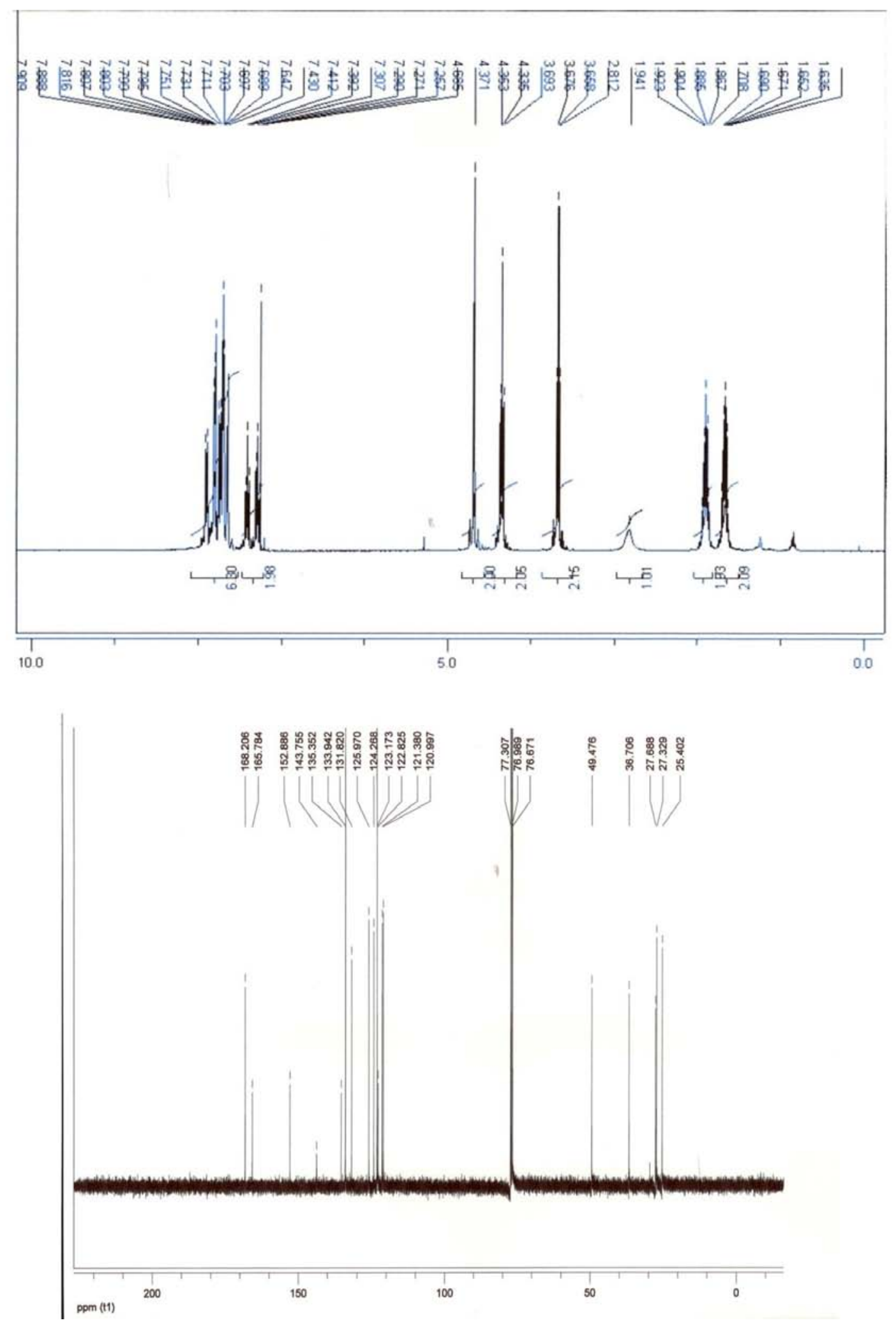

Figure S7. ${ }^{1} \mathrm{H}(400 \mathrm{MHz})$ and ${ }^{13} \mathrm{C}(100 \mathrm{MHz}) \mathrm{NMR}$ spectrum of compound $4 \mathbf{a}$ in $\mathrm{CDCl}_{3}$. 

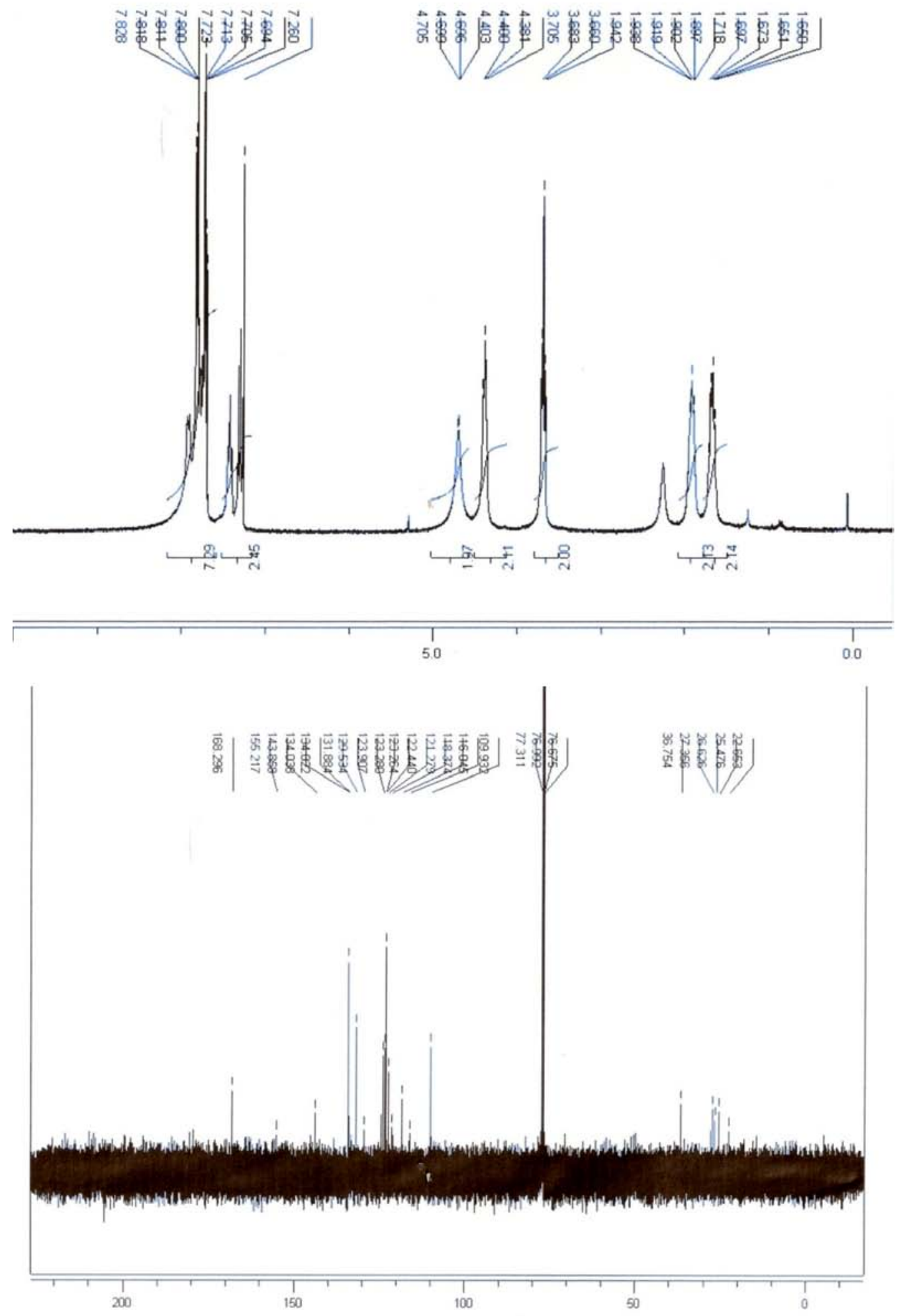

Figure S8. ${ }^{1} \mathrm{H}(300 \mathrm{MHz})$ and ${ }^{13} \mathrm{C}(75.5 \mathrm{MHz}) \mathrm{NMR}$ spectrum of compound $\mathbf{4 b}$ in $\mathrm{CDCl}_{3}$. 


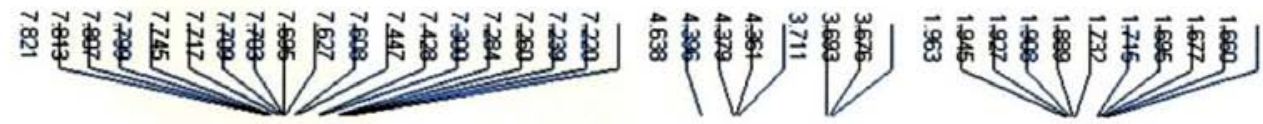
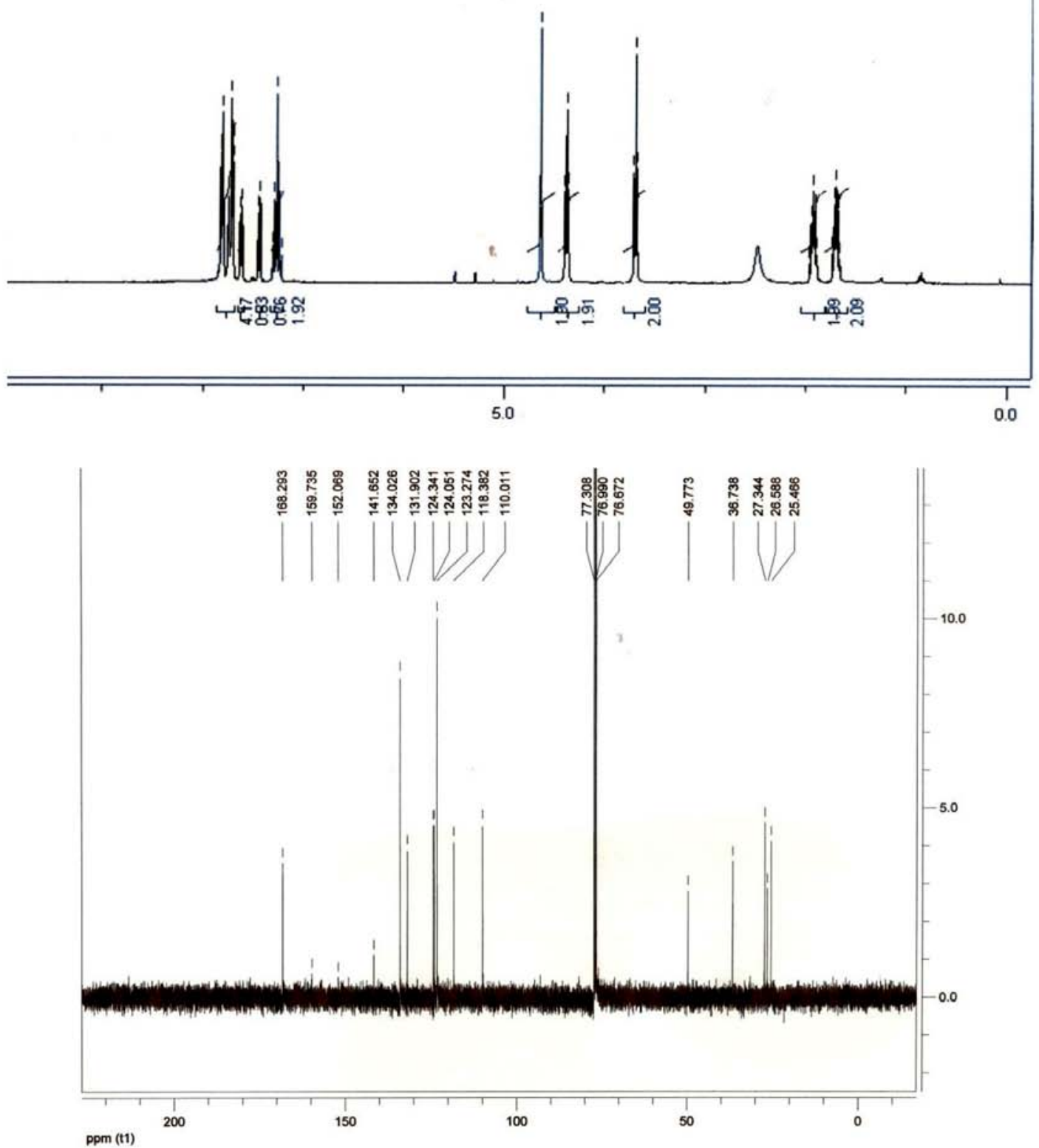

Figure S9. ${ }^{1} \mathrm{H}(400 \mathrm{MHz})$ and ${ }^{13} \mathrm{C}(100 \mathrm{MHz}) \mathrm{NMR}$ spectrum of compound $\mathbf{4 c}$ in $\mathrm{CDCl}_{3}$. 

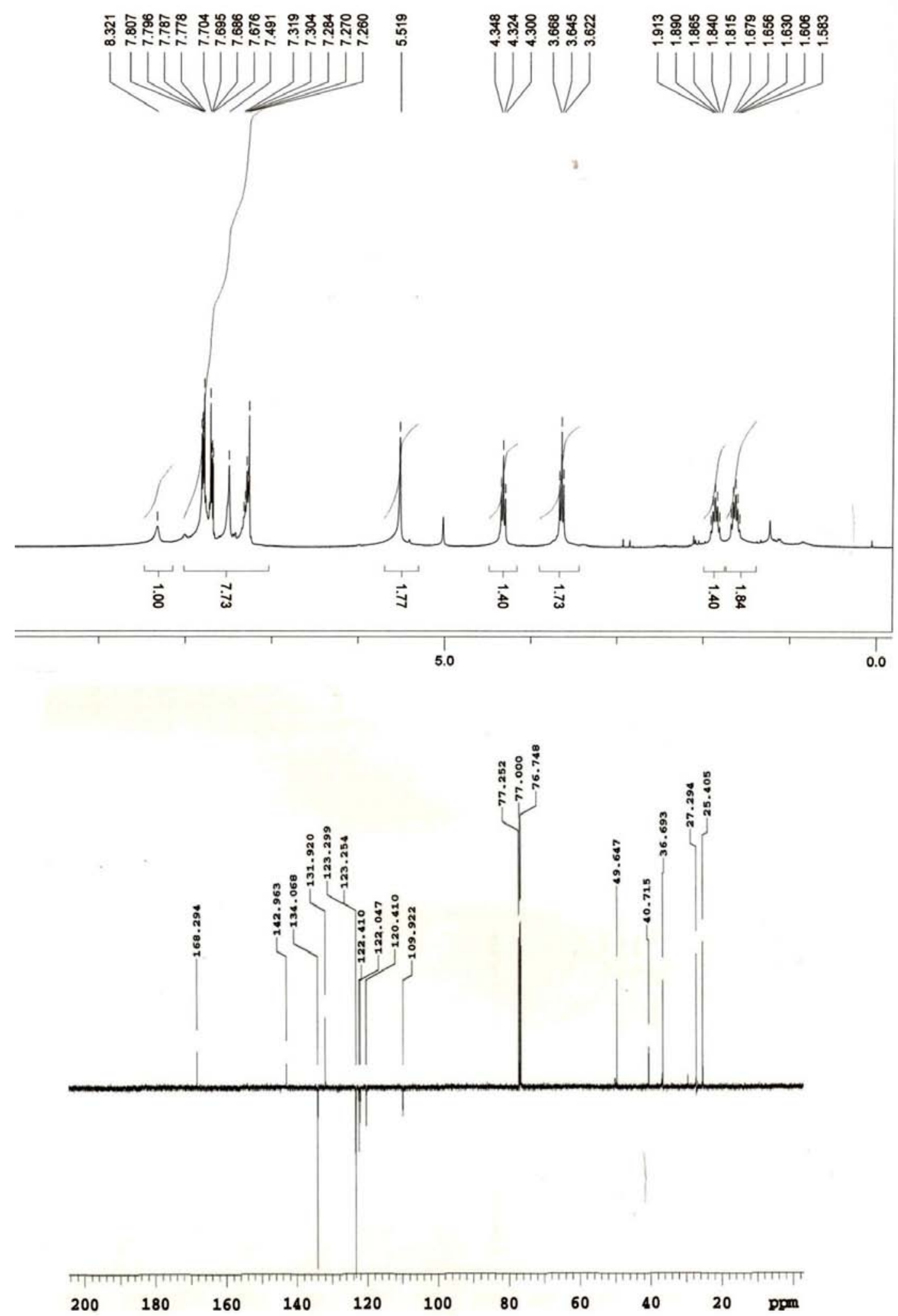

Figure S10. ${ }^{1} \mathrm{H}(300 \mathrm{MHz})$ and ${ }^{13} \mathrm{C}-\mathrm{APT}(125 \mathrm{MHz})$ NMR spectrum of compound $\mathbf{4 d} \mathrm{CDCl}_{3}$. 

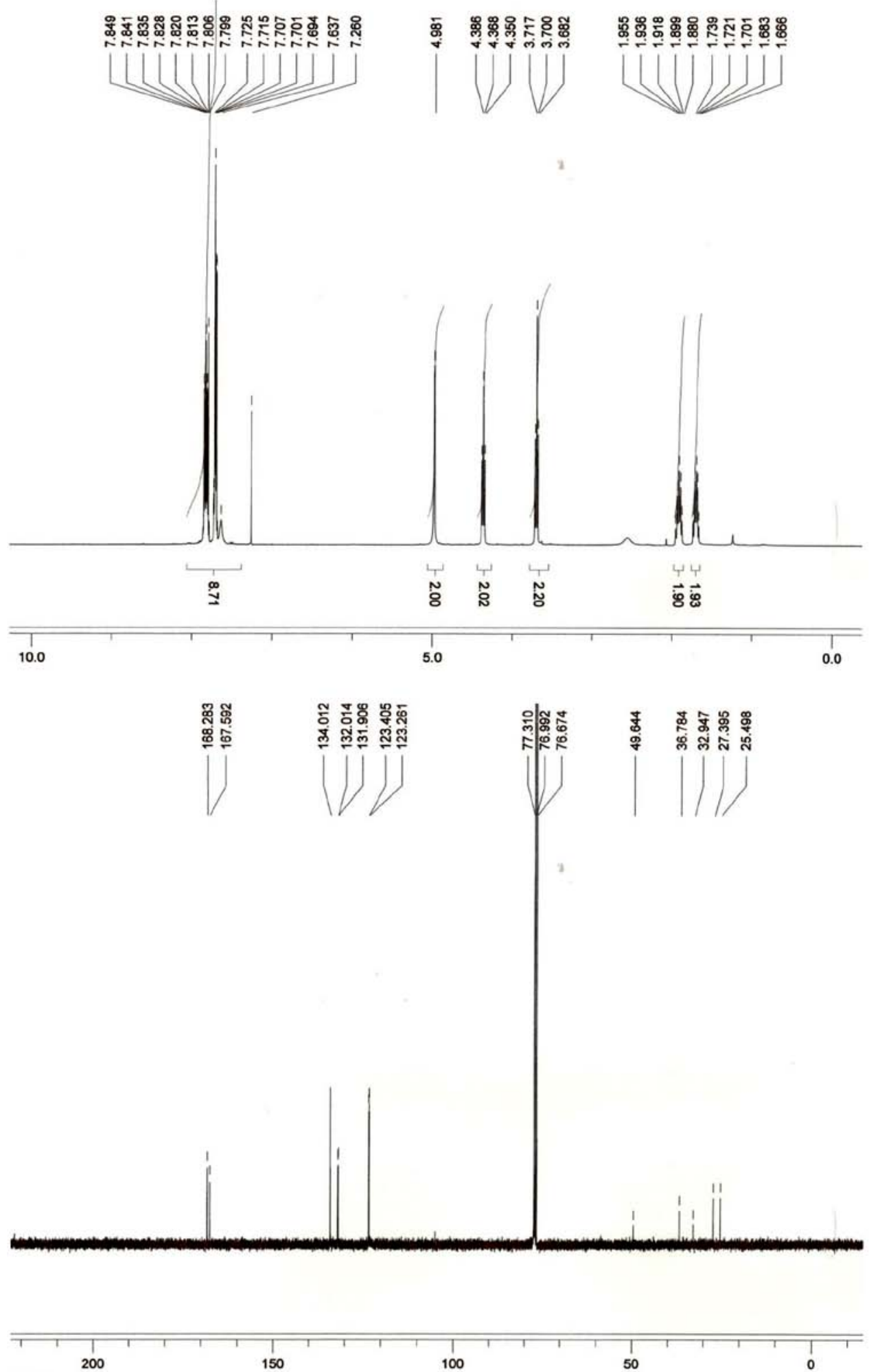

Figure S11. ${ }^{1} \mathrm{H}(400 \mathrm{MHz})$ and ${ }^{13} \mathrm{C}(100 \mathrm{MHz}) \mathrm{NMR}$ spectrum of compound $4 \mathrm{e} \mathrm{CDCl}_{3}$. 

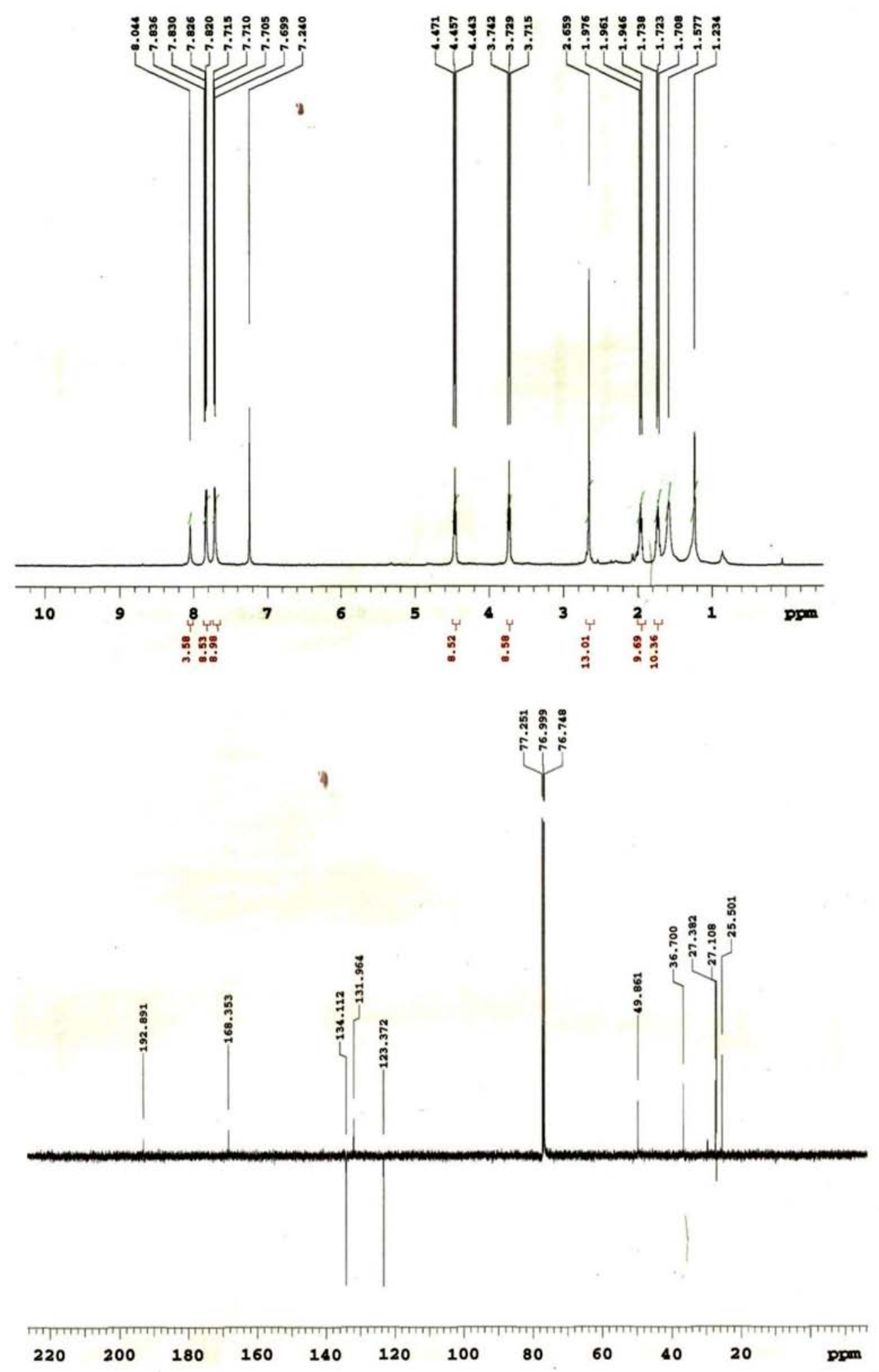

Figure S12. ${ }^{1} \mathrm{H}(500 \mathrm{MHz})$ and ${ }^{13} \mathrm{C}-\mathrm{APT}(125 \mathrm{MHz}) \mathrm{NMR}$ spectrum of compound $\mathbf{4 f}$ in $\mathrm{CDCl}_{3}$. 


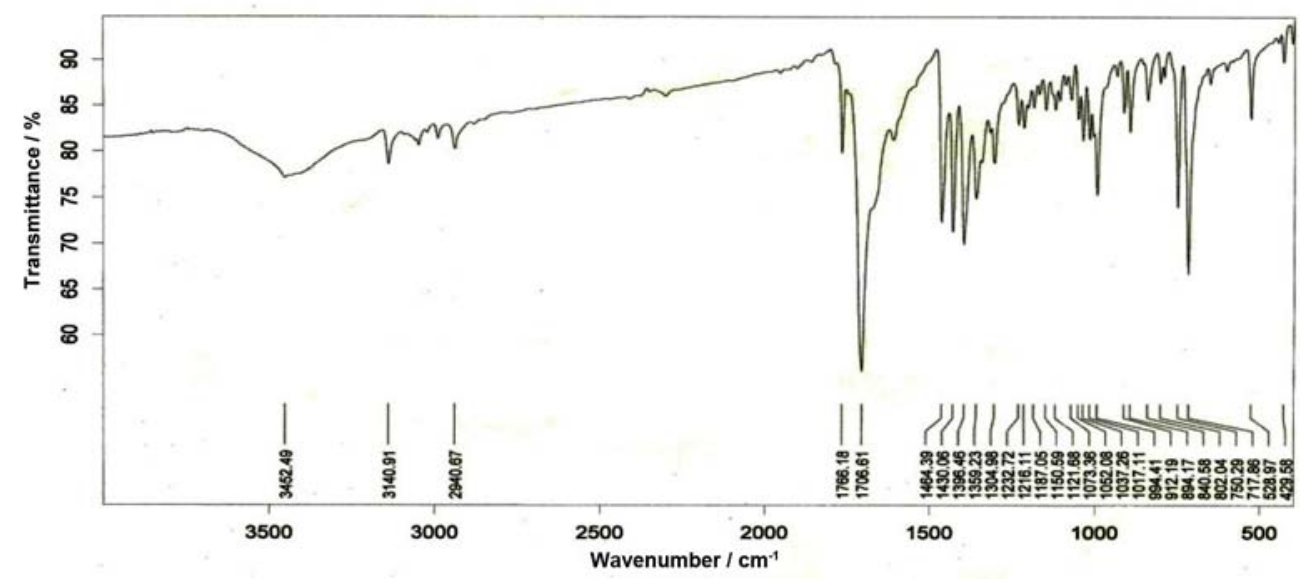

Figure S13. IR spectrum of compound 3a.

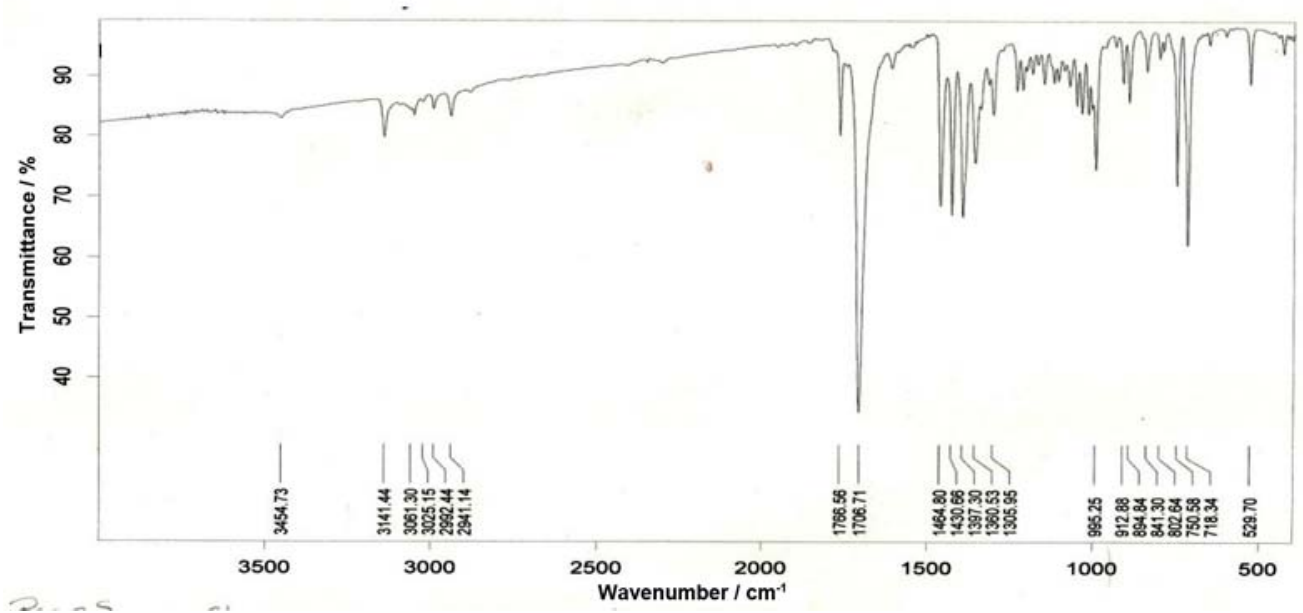

Figure S14. IR spectrum of compound $3 b$.

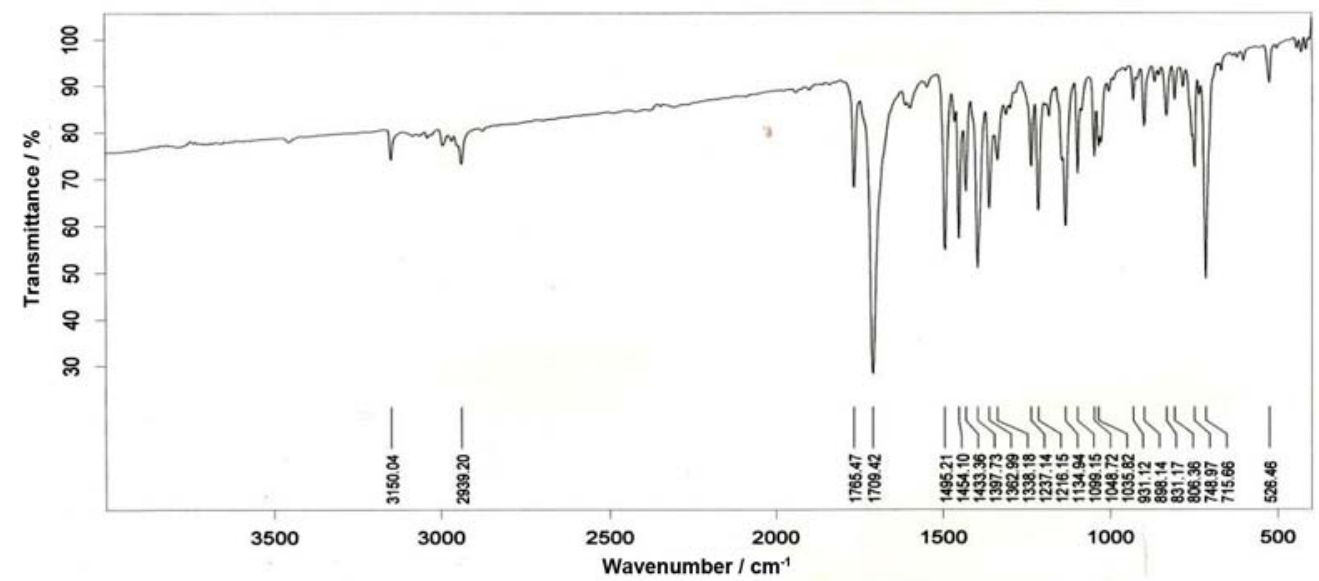

Figure S15. IR spectrum of compound 3c. 


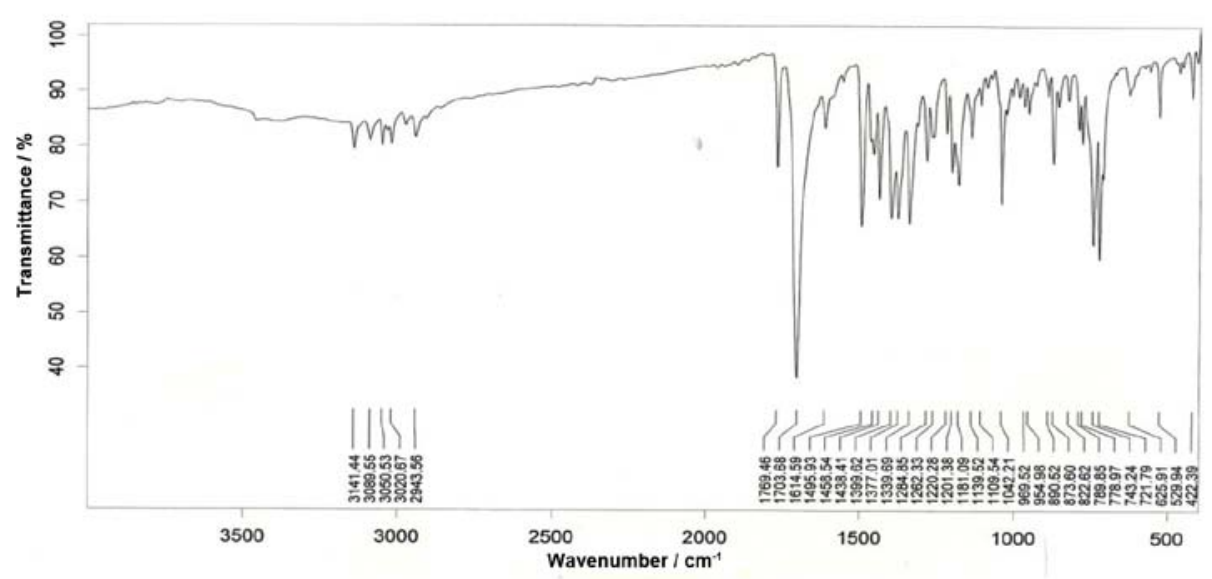

Figure S16. IR spectrum of compound 3d.

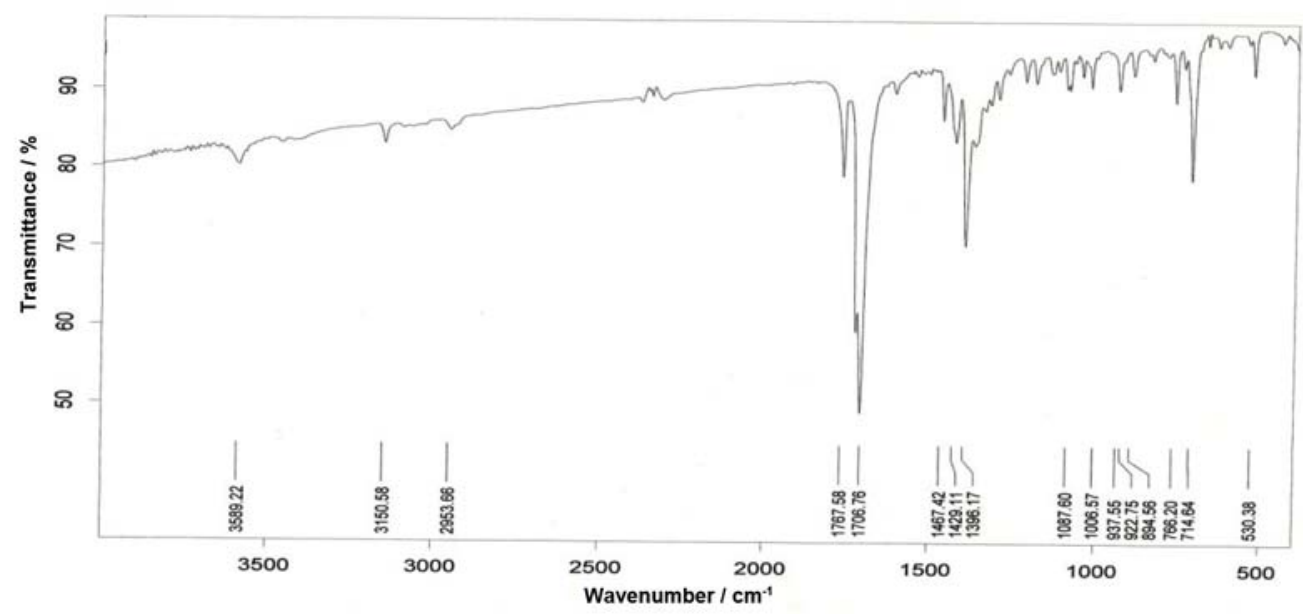

Figure S17. IR spectrum of compound 3e.

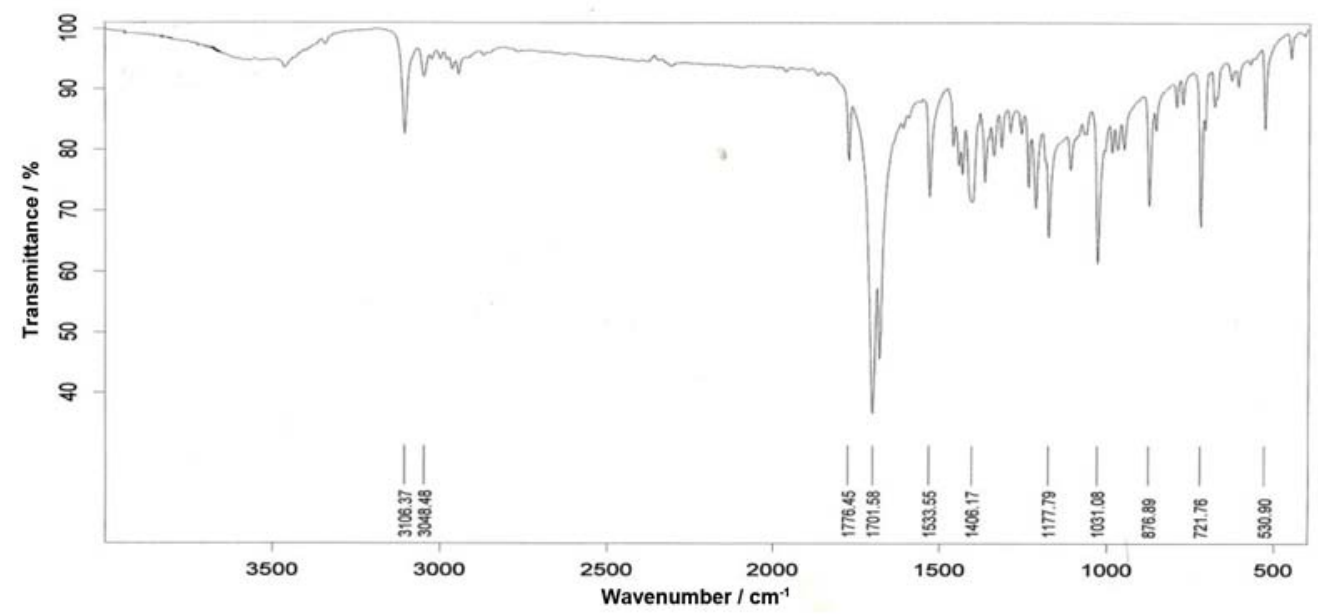

Figure S18. IR spectrum of compound 3 f. 


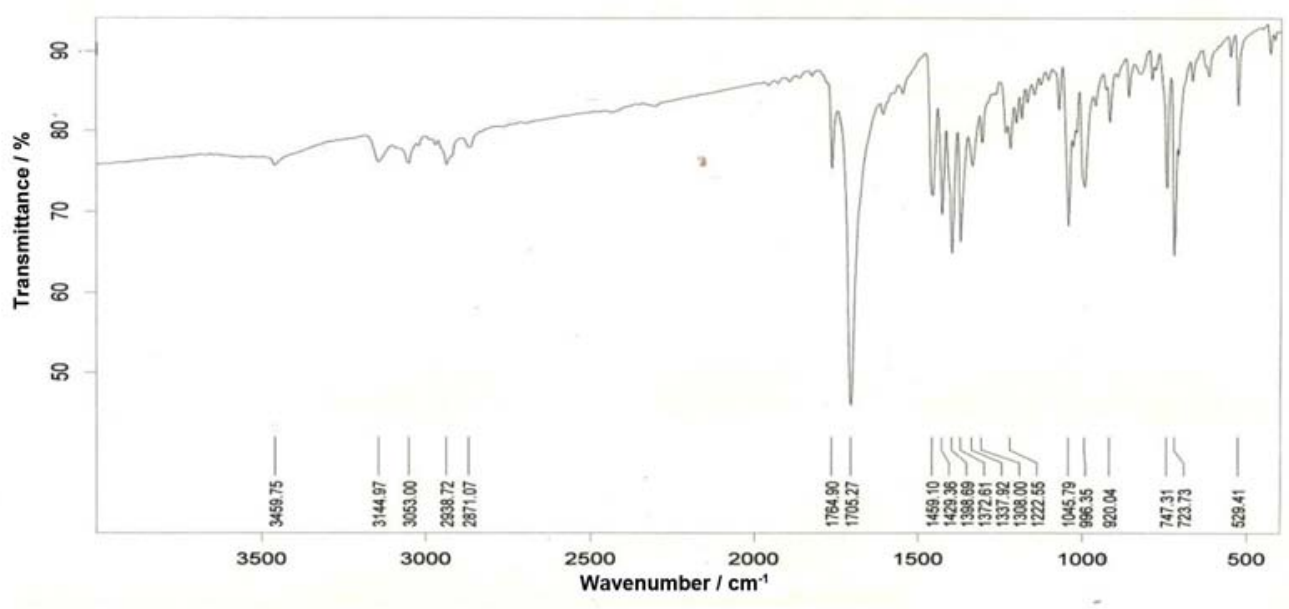

Figure S19. IR spectrum of compound $4 \mathbf{a}$.

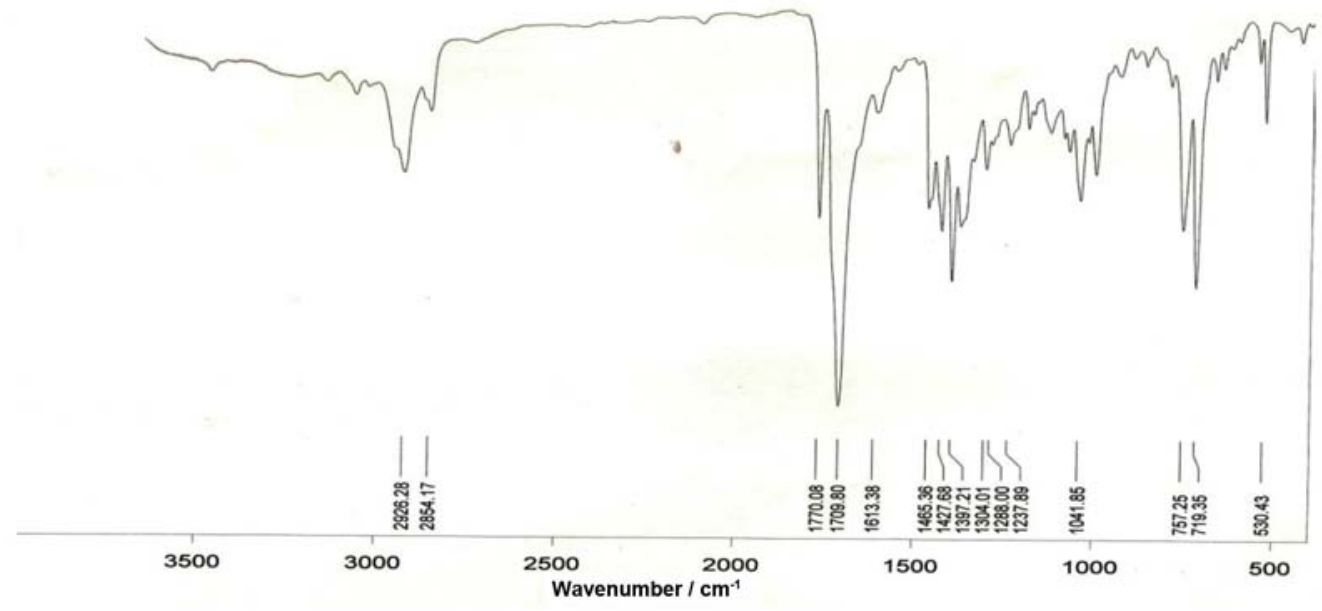

Figure S20. IR spectrum of compound $\mathbf{4 b}$.

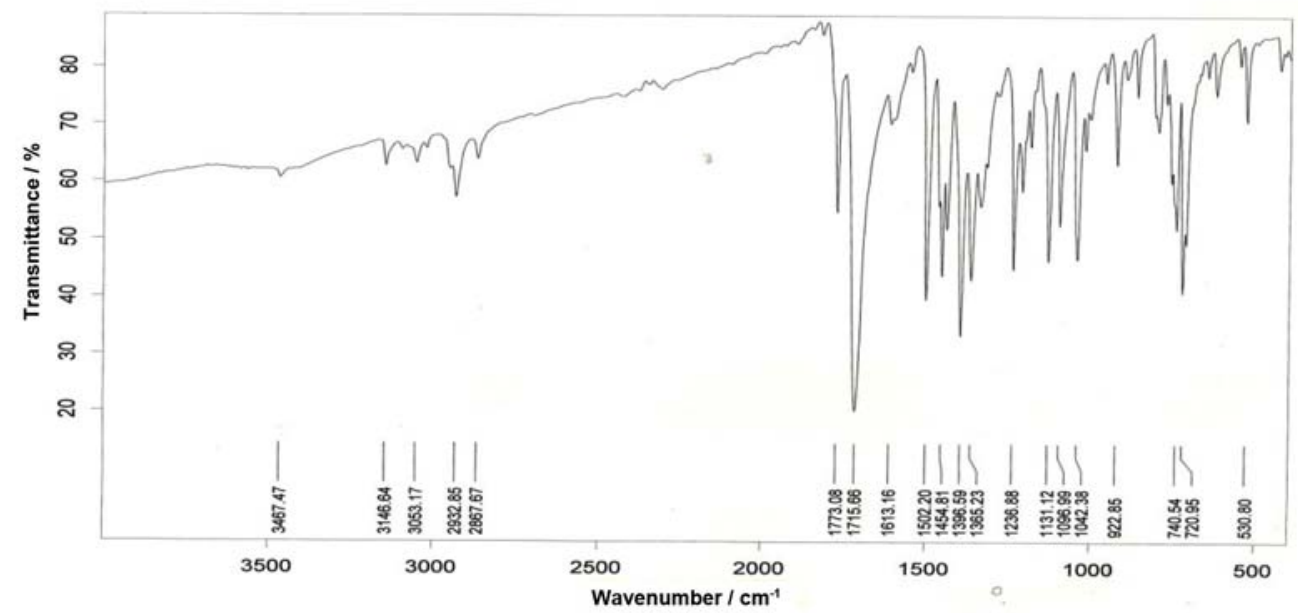

Figure S21. IR spectrum of compound $\mathbf{4 c}$. 


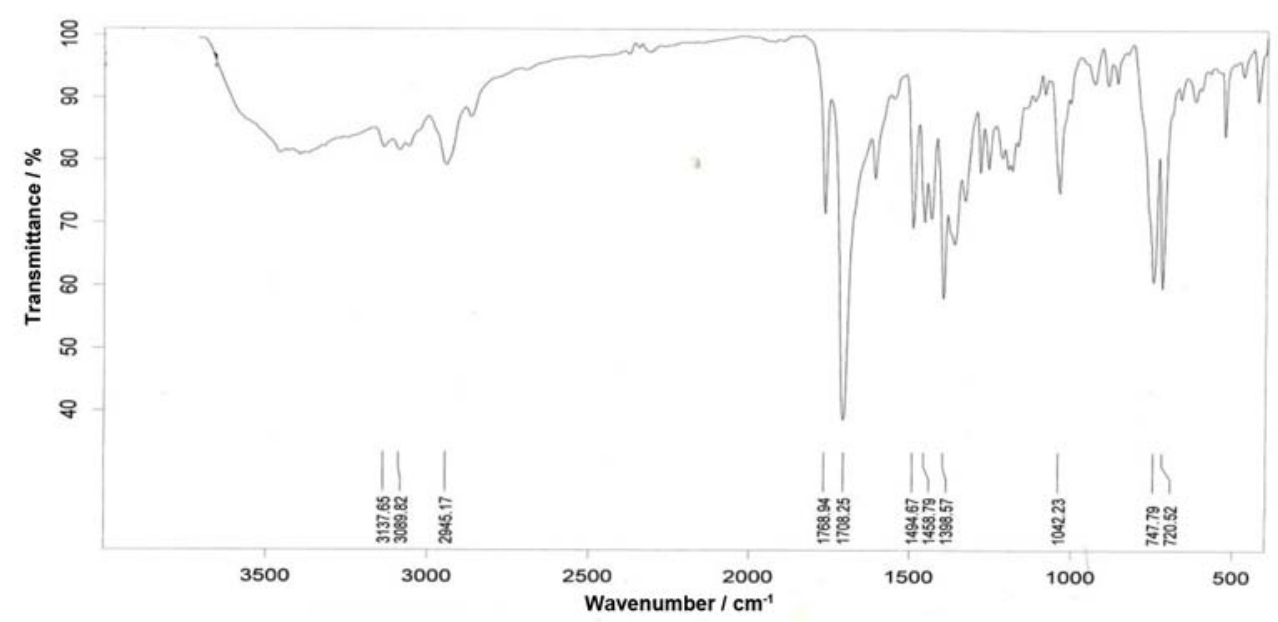

Figure S22. IR spectrum of compound 4 d.

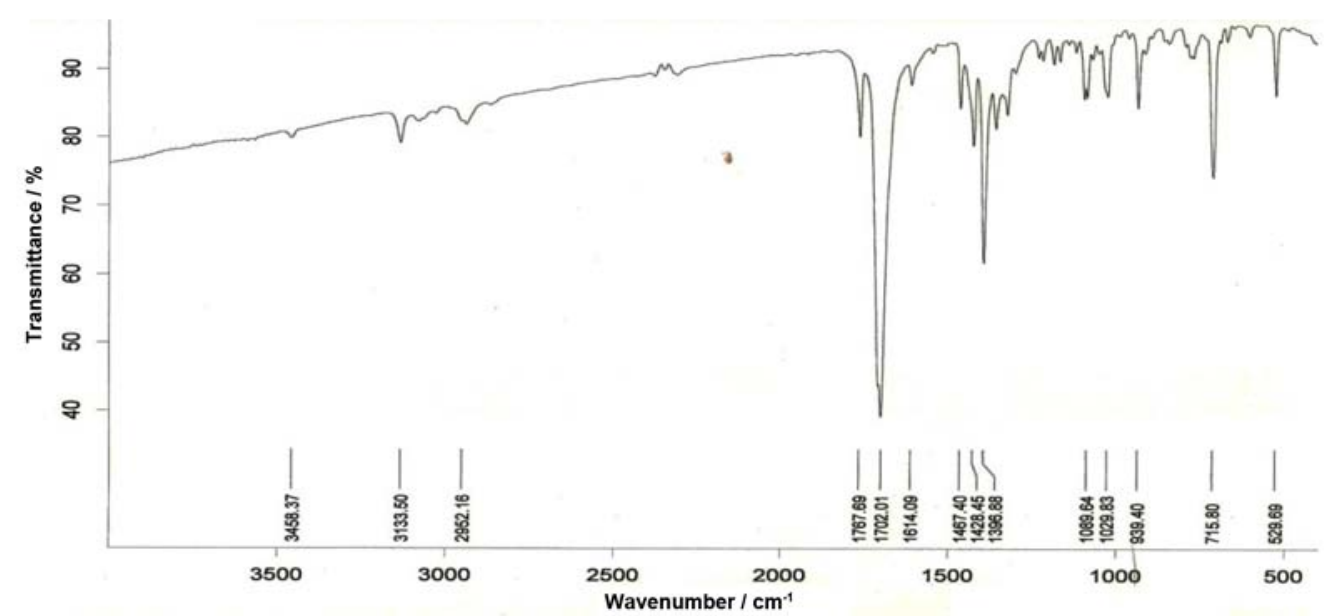

Figure S23. IR spectrum of compound 4e.

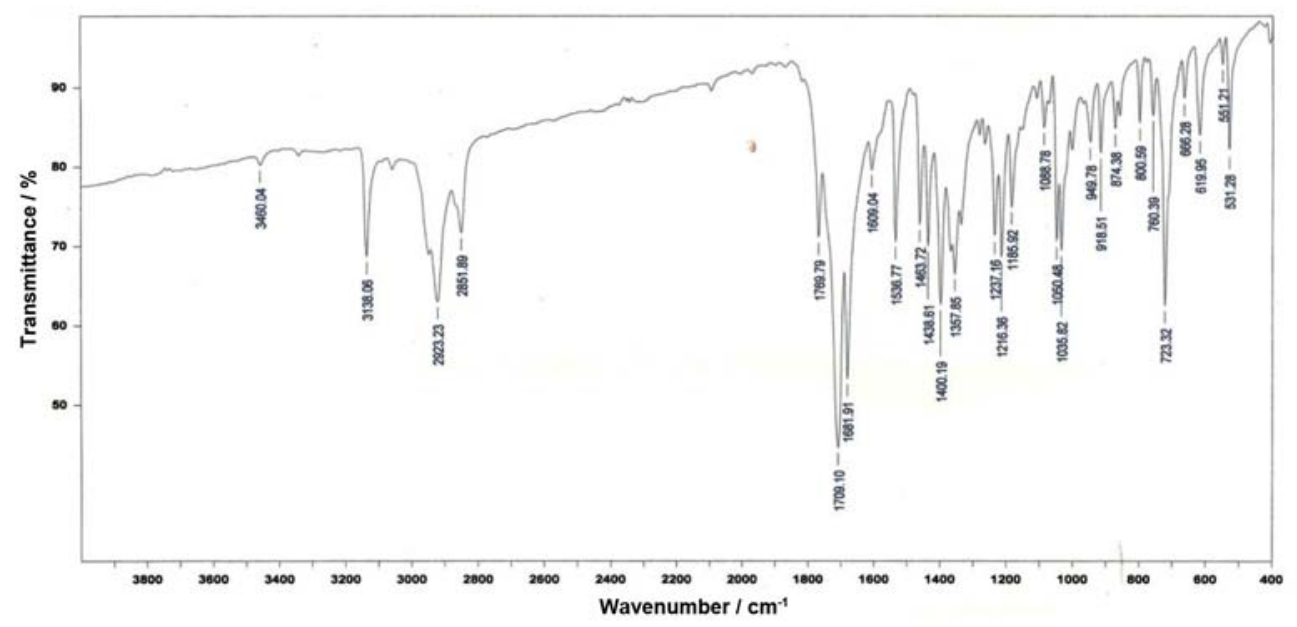

Figure S24. IR spectrum of compound $\mathbf{4 f}$. 

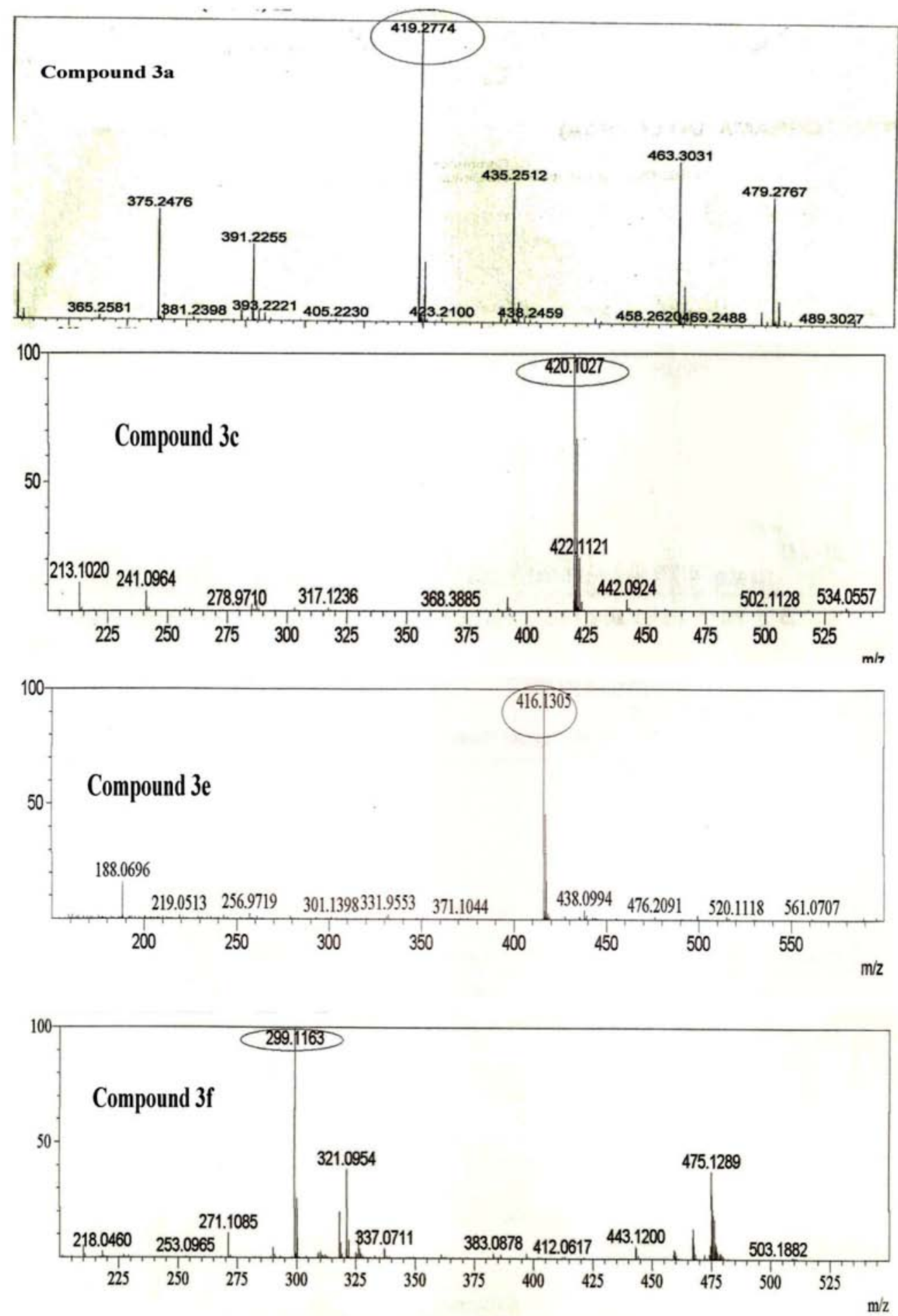

Figure S25. $m / z$ LC-MS spectrum graph of the compounds $\mathbf{3 a}, \mathbf{3 c}, \mathbf{3 e}$ and $\mathbf{3 f}$. 

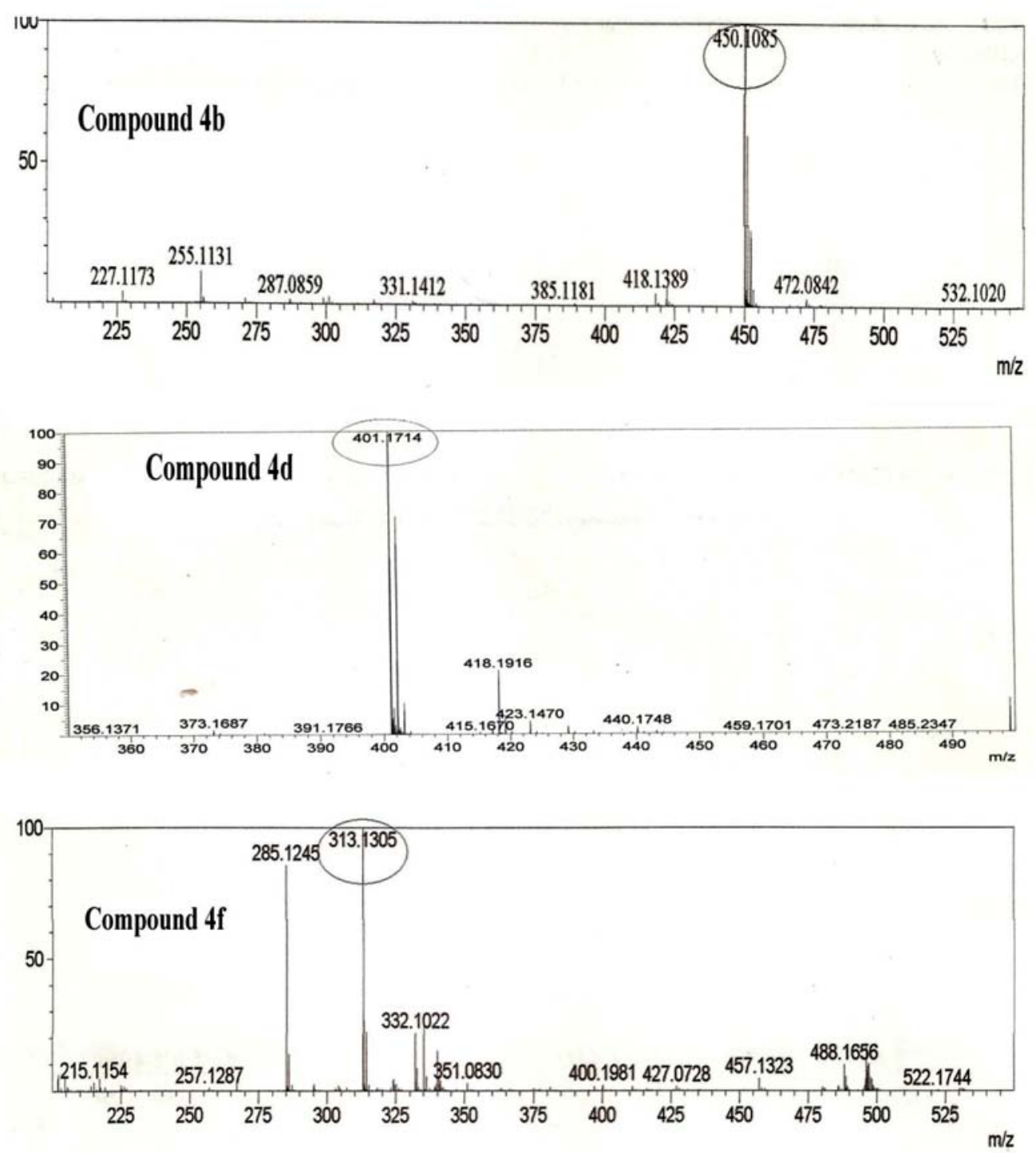

Figure S26. $m / z$ LC-MS spectrum graph of the compounds $\mathbf{4 b}, \mathbf{4 d}$ and $\mathbf{4 f}$. 